\title{
Decay Properties and Quasi-stationary Distributions for Stopped Markovian Bulk-Arrival and Bulk-Service Queues
}

\author{
CHEN ANYUE* University of Liverpool and Xi'an Jiaotong-Liverpool University \\ LI JUNPING** Central South University \\ HOU ZHENTING *** Central South University \\ NG KAI WANG ${ }^{* * * *}$ University of Hong Kong
}

\begin{abstract}
We consider decay properties including the decay parameter, invariant measures and quasistationary distributions for a Markovian bulk-arrival and bulk-service queue which stops when the waiting line is empty. Investigating such a model is crucial for understanding the busy period and other related properties of the Markovian bulk-arrival and bulk-service queuing processes. The exact value of the decay parameter $\lambda_{C}$ is firstly obtained. We show that the decay parameter can be easily expressed explicitly. The invariant measures and quasi-distributions are then revealed. We show that there exists a family of invariant measures indexed by $\lambda \in\left[0, \lambda_{C}\right]$. We then show that under some mild conditions, there exists a family of quasi-stationary distributions also indexed by $\lambda \in\left[0, \lambda_{C}\right]$. The generating functions of these invariant measures and quasi-stationary distributions are presented. We further show that this stopped Markovian bulk-arrival and bulk-service queueing model is always $\lambda_{C}$-transient. Some deep properties regarding $\lambda_{C}$-transience are examined and revealed. The clear geometric interpretation of the decay parameter is explained. A few examples are then provided to illustrate the results obtained in this paper.
\end{abstract}

Keywords: Stopped Markovian bulk-arrival and bulk-service queues; Decay parameter; Invariant measures; Quasi-stationary distributions.

AMS 2000 SUBJECT CLASSIFICATION: PRIMARY 60J27

SECONDARY 60J35, 60J80

\section{Introduction}

During the development of general theory and applications of continuous time Markov chains, there is a long history in investigating a few closely linked and very important con-

\footnotetext{
* Postal address: Division of Statistics and Probability, Department of Mathematical Sciences, The University of Liverpool, Liverpool, L69 7ZL, UK. E-mail address: achen@liv.ac.uk and Department of mathematics, Xi'an Jiaotong-Liverpool University, Suzhou, 215123, China.

** Postal address: School of Mathematical Science and Computing Technology, Central South University, Changsha, 410075, China. E-mail address: jpli@mail.csu.edu.cn

*** Postal address: School of Mathematical Science and Computing Technology, Central South University, Changsha, 410075, China. E-mail address: zthou@mail.csu.edu.cn

**** Postal address: Department of Statistics and Actuarial Science, University of Hong Kong, Pokfulam Road, Hong Kong, HKSAR. E-mail address: kaing@hku.hk
} 
cepts: the decay parameter, invariant measures, invariant vectors, and the quasi-stationary distributions. While the idea of using a quasi-stationary distribution can be traced back at least to the early work of Yaglom [45], who considered the long run behavior, in the sense of conditional distribution, of the subcritical Galton-Watson process, the key feature and importance of this theory was not fully realised and explored until the publication of the remarkable work of Vere-Jones [44]. At nearly the same time, the existence of the decay parameter was firstly revealed by Kingman, when he showed in Kingman [25] that if $C$ is an irreducible class of a continuous time Markov chain defined on the state space $\mathbf{E}$, then there exists a number $\lambda_{C} \geq 0$, called the decay parameter of the corresponding process, such that for all $i, j \in C$,

$$
\frac{1}{t} \log p_{i j}(t) \rightarrow-\lambda_{C} \text { as } t \rightarrow+\infty,
$$

where $P(t)=\left(p_{i j}(t) ; i, j \in \mathbf{E}\right)$ is the transition function of the corresponding continuous time Markov chain. It can be easily proved that this decay parameter can be expressed as

$$
\lambda_{C}=\inf \left\{\lambda \geq 0: \int_{0}^{\infty} e^{\lambda t} p_{i j}(t) d t=\infty\right\}=\sup \left\{\lambda \geq 0: \int_{0}^{\infty} e^{\lambda t} p_{i j}(t) d t<\infty\right\}
$$

where the quantities in the middle and right hand side of (1.1) are independent of $i, j \in C$. Beginning with this pioneer and remarkable work, this extremely useful theory has been flourished due to much important research, including the significant contributions made by Flaspohler [16], Pollett [36], Darroch and Seneta [13], Kelly [23], Kijima [24], Nair and Pollett [32], Tweedie [41], Van Doorn [42], [43] and many others.

On the other hand, Markovian queueing theory, as one of the most notable and prominent as well as one of the oldest subareas in applied stochastic processes, occupies a significant niche in applied probability. Markovian queueing theory plays an important role both in the development of general queueing models (for example, Asmussen [4], Gross and Harris [19], Kleinrock [26] and Medhi [31]) and in the theory and application of continuoustime Markov chains (for example, Anderson [1] and Chung [12]). Within this framework, state-dependent input and output mechanisms have attracted considerable attention. In particular, Gelenbe [17] and Gelenbe, Glynn and Sigman [18] introduced the very useful concept of negative arrival, and this was followed up by other authors, including Bayer and Boxma [5], Harrison and Pitel [20], Henderson [21] and Jain and Sigman [22]. The queueing models with negative arrivals also has close theoretical links with the versatile Markovian arrival processes introduced by Neuts [33], which include several kinds of batcharrival process. Additionally, Neuts [34] described a number of interesting batch-arrival models together with useful methods for analysing them. For further developments, see Stadje [38], Lucantoni [29], Lucantoni and Neuts [30], Nishimura and Sato [35] and Dudin and Nishimura [14]. Also, in Chen and Renshaw [10,11] the possibility of clearing the entire workload is allowed. At the same time, the theory of bulk queues (bulk arrival and/or bulk service) is also well developed. See the excellent reference of Chaudhry and Templeton [7]. For recent advances see Armero and Conesa [2], Arumuganathan and Ramaswami [3], Chang, Choi and Kim [6], Fakinos [15], Srinivasan, Renganathan and Kalyanaraman [37], Sumita and Masuda [39] and Ushakumari and Krishnamoorthy [40]. The queueing models with both bulk-arrival and bulk-service incorporating with state-dependent control have also been attracted much interest. In particular, very recently, Chen et al [8] discussed an 
interesting model where state-dependent control can happen only when no customer is at waiting list except possibly a customer in service.

In spite of these developments in both topics, it seems that the deep relationship between decay properties and Markovian queueing models has not been fully revealed. In fact, even calculating the exact value of the decay parameter for most transient Markovian queueing models is still an open problem. In particular, we still know little about decay properties of the queueing model discussed in Chen et al [8]. Note that, however, if the bulk-service is not presented, the decay parameter and the associated properties have been examined in Li and Chen $[27,28]$.

The main aim of this paper is therefore to consider the decay parameter and the associated properties including the most important invariant measure and quasi-stationary distributions of the Markovian bulk-arrival and bulk-service queue with state-dependent control explored in Chen $[27,28]$. Investigating such properties will greatly deepen our understanding of the busy period distribution of the corresponding queueing model and provide much information in designing appropriate state-dependent control, which, in turn, could significantly improve the efficiency and effectiveness of the queueing systems. We shall see that this basic aim has been achieved in this paper. In particular, we shall show that the decay parameter for our models can be obtained very easily. Many deep associated properties have also been revealed. Based on the results obtained in this paper, we shall be able to further investigate the decay parameter and the associated properties for the more general Markovian bulk-arrival and bulk-service queue with state-dependent controls, particularly for the transient case, in our subsequent papers,

In addition to achieving the above aims, this paper is also of methodological significance. Indeed, several new ideas and methods are employed in our analysis. These new methods will be very useful in analyzing more complex models. This will also be revealed in our subsequent papers.

We now begin our study by specifying our known conditions. That is that we first specify the generator matrix, i.e., the so-called $q$-matrix $Q=\left(q_{i j} ; i, j \in \mathbf{Z}_{+}\right)$, of our stopped Markovian bulk-arrival and bulk-service queue queueing models, where $\mathbf{Z}_{+}$stands for the nonnegative integers $\{0,1,2, \cdots\}$.

Definition 1.1. A $q$-matrix $Q=\left(q_{i j} ; i, j \in \mathbf{Z}_{+}\right)$is called a stopped bulk-arrival and bulkservice generator (henceforth referred to as a stopped BABS generator), if

$$
q_{i j}= \begin{cases}b_{j-i+2}, & \text { if } i \geq 2, j \geq i-2 \\ 0, & \text { otherwise }\end{cases}
$$

where

$$
b_{0}>0, b_{j} \geq 0(j \neq 2), 0<\sum_{j \neq 2} b_{j} \leq-b_{2}<\infty .
$$

In order to avoid discussing some trivial cases, we shall, throughout this paper, assume that

$$
\sum_{j=3}^{\infty} b_{j}>0
$$


and

$$
\sum_{j=0}^{\infty} b_{2 j+1}>0
$$

Also, let

$$
m_{d}=2 b_{0}+b_{1}, \quad m_{b}=\sum_{j=3}^{\infty}(j-2) b_{j}
$$

denote the mean service rate and the mean arrival rate, respectively.

By (1.2) we may see that the behaviour of the stopped BABS could be described as follows. Whenever the queuing length is great than 1 , then the arrival of customers follows a compound Poisson process with arrival of a batch of $r$ individuals with rate $b_{r+2}(r \geq$ 1) coupled with batch service to $j$ individuals with rate $b_{2-j}(j=1,2)$. The queueing process will stop moving whenever either the queue is empty or there is only one customer being-served in the queue. From practical point of view, this is, of course, not a realistic model. However, as shown in Chen et al [11], this structure is the underlined structure of a Markovian bulk-arrival and bulk-service queue with state-dependent control and thus plays extremely important role in analysis the latter practical model.

By (1.3) we see that non-conservativeness is allowed in this paper. The reason for dealing with this general definition is two-fold: firstly in our later proof, we need to consider the nonconservative case. Secondly and more importantly, this definition provides us an opportunity to consider more general models.

Let

$$
d=-\sum_{j=0}^{\infty} b_{j}
$$

be the deficit. Then $d \geq 0$ and $d=0$ if and only if $Q$ is conservative.

Definition 1.2. Let $Q=\left(q_{i j} ; i, j \in \mathbf{Z}_{+}\right)$be a stopped BABS generator matrix defined in (1.2)-(1.3). The corresponding transition function $P(t)=\left(p_{i j}(t) ; i, j \in \mathbf{Z}_{+}\right)$, usually called the $Q$-function, is called a stopped Markovian bulk-arrival and bulk-service queueing process (henceforth referred to as a stopped BABS-process).

Note that we have defined the queueing process as the corresponding transition function $P(t)$ rather than the process itself. In fact, for convenience, we shall freely use this term to denote either of them in this paper. Also, under this interpretation, the terms $Q$-process and $Q$-function, referring to a specific $q$-matrix $Q$, are identical. Such kind of usage is, of course, commonly accepted and won't cause any confusion. Since our generator matrix $Q$ is bounded and thus, by the general theory of continuous time Markov chains, we know that there exists only one $Q$-process which is the Feller minimal one. Since we have assume that both $b_{0}>0$ and (1.4)-(1.5) hold, it is clear that $C=\{2,3, \cdots\}$ is an irreducible class for $Q$ and, also, for the corresponding $Q$-function $P(t)=\left(p_{i j}(t) ; i, j \in \mathbf{Z}_{+}\right)$.

It is well known that the decay parameter and quasi-stationary distributions are closely linked with the so-called $\mu$-subinvariant measures and $\mu$-subinvariant vectors. An elementary but detailed discussion of this theory can be seen in Anderson [1]. For convenience, we briefly repeat these definitions, tailored for our special models, as follows: 
Definition 1.3. Let $Q=\left(q_{i j} ; i, j \in \mathbf{Z}_{+}\right)$be a stable generator matrix and $C$ be a communicating class of $\mathbf{Z}_{+}$. Assume that $\mu \geq 0$. A set $\left(m_{i} ; i \in C\right)$ of strictly positive numbers is called a $\mu$-subinvariant measure for $Q$ on $C$ if

$$
\sum_{i \in C} m_{i} q_{i j} \leq-\mu m_{j}, \quad j \in C
$$

If equality holds in (1.8), then $\left(m_{i} ; i \in C\right)$ is called a $\mu$-invariant measure for $Q$ on $C$.

Definition 1.4. Let $P(t)=\left(p_{i j}(t) ; i, j \in \mathbf{Z}_{+}\right)$be a $Q$-function and $C$ is a communicating class of $\mathbf{Z}_{+}$. Assume that $\mu \geq 0$. A set $\left(m_{i} ; i \in C\right)$ of strictly positive numbers is called a $\mu$-subinvariant measure for $P(t)=\left(p_{i j}(t) ; i, j \in \mathbf{Z}_{+}\right)$if

$$
\sum_{i \in C} m_{i} p_{i j}(t) \leq e^{-\mu t} m_{j}, \quad j \in C .
$$

If equality holds in (1.9), then $\left(m_{i} ; i \in C\right)$ is called a $\mu$-invariant measure for $P(t)$ on $C$.

The subinvariant vectors can be similarly defined. Finally, following Van Doorn [43], we give the definition of quasi-stationary distributions as follows.

Definition 1.5. Suppose that $P(t)=\left(p_{i j}(t) ; i, j \in \mathbf{Z}_{+}\right)$is a $Q$-function. Assume that $C$ is a communicating class of $\mathbf{Z}_{+}$and $\left(m_{i} ; i \in C\right)$ is a probability distribution over $C$. Let $p_{j}(t)=\sum_{i \in C} m_{i} p_{i j}(t)$, for $j \in C$ and $t \geq 0$. If

$$
\frac{p_{j}(t)}{\sum_{i \in C} p_{i}(t)}=m_{j}, \quad j \in C, t>0
$$

then $\left(m_{i} ; i \in C\right)$ is called a quasi-stationary distribution.

The relationship between invariant measures and quasi-stationary distributions has been revealed by, say, the work of Van Doorn [43] and Nair and Pollett [32].

The structure of this paper is as follows. Some preliminary results, which also provide useful techniques and methods to be used later, will be firstly presented in Section 2. In Section 3 we concentrate on studying the decay parameter for the stopped Markovian bulkarrival and bulk-service queues. The exact value of decay parameter will be revealed in two theorems which deal with two different cases by using different approaches. The simple and practical calculation methods are then presented. The interesting geometric interpretation is also explained in this section. Transience properties related to the decay parameter will be discussed in the following Section 4. We shall prove that a stopped Markovian bulkarrival and bulk-service queueing process is always $\lambda_{C}$-transient and some exact expressions closely related to this property are revealed. The important $\lambda_{C}$-invariant measures and quasi-stationary distributions are addressed in Section 5. We show that there exists a family of invariant measures indexed by $\lambda \in\left[0, \lambda_{C}\right]$. We then show that under some mild conditions, there exists a family of quasi-stationary distributions also indexed by $\lambda \in\left[0, \lambda_{C}\right]$. The generating functions of these invariant measures and quasi-stationary distributions are presented. In the final section 6, several examples are provided to illustrate the results obtained in the previous sections.

\section{Preliminaries}


In order to find the decay parameter $\lambda_{C}$ and to study invariant measures and quasistationary distributions for the stopped BABS-processes, we need to define a function $B(s)$ which is the generating function of $\left\{b_{k} ; k \geq 0\right\}$, i.e.,

$$
B(s)=\sum_{k=0}^{\infty} b_{k} s^{k} .
$$

with, as a power series, convergence radius

$$
\rho=1 / \limsup _{n \rightarrow \infty} \sqrt[n]{b_{n}}
$$

Clearly,

$$
\rho \geq 1
$$

By Noting that $B(0)=b_{0}>0$ and $B(1)=-d \leq 0$, we know that $B(s)=0$ has a root $q_{0} \in(0,1]$. Also $B(1)=0$ if and only if $d=0$.

Our first observation on $B(s)$ is that both $B(s)$ and $B^{\prime}(s)$ are strictly piecewise monotone functions on $[0, \rho)$ with at most two interlaced nonnegative zeros, respectively. In fact, $B^{\prime}(s)$ is even convex on $[0, \rho)$, as the following two lemmas show.

Lemma 2.1. Assume that $\rho=+\infty$. Then $B(s)$ is a piecewise convex and monotone function on $[0, \infty)$ with exactly two zeros $0<q_{S}<q_{L}<\infty$, while $B^{\prime}(s)$ is a convex function on $[0, \infty)$ with exactly two zeros $0 \leq \xi_{S}<\xi_{L}<\infty$. More specifically,

(i) if $d=0$ and $m_{d}=m_{b}$ (or equivalently, $B(1)=B^{\prime}(1)=0$ ), then there exist four points $0 \leq \xi_{S}<q_{S}=\xi_{L}=q_{L}=1$ such that $B^{\prime}\left(\xi_{S}\right)=B^{\prime}\left(\xi_{L}\right)=B\left(q_{S}\right)=B\left(q_{L}\right)=0$. Moreover, if $b_{1}=0$ then $\xi_{S}=0$ and $B^{\prime}(s)<0$ for $s \in(0,1)$ and $B^{\prime}(s)>0$ for $s \in(1, \infty)$ and that $B(s)>0$ for all $s \geq 0$ except that $B(1)=0$, while if $b_{1}>0$ then $0<\xi_{S}<1$, and that $B^{\prime}(s)<0$ for $s \in\left(\xi_{S}, 1\right)$ and $B^{\prime}(s)>0$ for $s \in\left(0, \xi_{S}\right) \cup(1, \infty)$ and that $B(s)>0$ for all $s \geq 0$ except that $B(1)=0$.

(ii) If $d>0$ or $m_{d} \neq m_{b}$, then there exist four interlaced points $0 \leq \xi_{S}<q_{S}<\xi_{L}<q_{L}<$ $+\infty$ such that $B^{\prime}\left(\xi_{S}\right)=B^{\prime}\left(\xi_{L}\right)=B\left(q_{S}\right)=B\left(q_{L}\right)=0$. Moreover, if $b_{1}=0$ then $\xi_{S}=0$ and that $B^{\prime}(s)<0$ for $s \in\left(0, \xi_{L}\right)$ and $B^{\prime}(s)>0$ for $s \in\left(\xi_{L}, \infty\right)$ and that $B(s)<0$ for $s \in\left(q_{S}, q_{L}\right)$ and $B(s)>0$ for $s \in\left[0, q_{S}\right) \cup\left(q_{L}, \infty\right)$. If $b_{1}>0$ then $\xi_{S}>0$, and that $B^{\prime}(s)<0$ for $s \in\left(\xi_{S}, \xi_{L}\right)$ and $B^{\prime}(s)>0$ for $s \in\left[0, \xi_{S}\right) \cup\left(\xi_{L}, \infty\right)$ and that $B(s)<0$ for $s \in\left(q_{S}, q_{L}\right)$ and $B(s)>0$ for all $s \in\left[0, q_{S}\right) \cup\left(q_{L}, \infty\right)$.

Proof. First note that the function

$$
B^{\prime \prime \prime}(s)=\sum_{j=3}^{\infty} j(j-1)(j-2) b_{j} s^{j-3}
$$

is positive for all $s>0$ (note also $B^{\prime \prime \prime}(0) \geq 0$ ), which is due to the assumption (1.4). It follows that $B^{\prime}(s)$ and $B^{\prime \prime}(s)$ are strictly convex and increasing functions, respectively, on $[0, \infty)$. Also, by the facts that $\rho=+\infty$ and (1.4), it is easily seen that all of $B^{\prime \prime \prime}(s), B^{\prime \prime}(s), B^{\prime}(s)$ and $B(s)$ tend to $+\infty$ as $s \rightarrow+\infty$. Considering that $B^{\prime \prime}(0)=2 b_{2}<0$ and $\lim _{s \rightarrow+\infty} B^{\prime \prime}(s)=+\infty$ and the fact that $B^{\prime \prime}(s)$ is strictly increasing on $[0, \infty)$, we know that $B^{\prime \prime}(s)$ has exactly one 
zero, denoted by $\eta$, on $(0, \infty)$ and that $B^{\prime \prime}(s)<0$ for $s \in[0, \eta)$ and $B^{\prime \prime}(s)>0$ for $s \in(\eta, \infty)$. Hence $B^{\prime}(s)$ is strictly decreasing on $[0, \eta)$ and strictly increasing on $[\eta, \infty)$.

Now, if $B^{\prime}(0)=b_{1}=0$, then 0 is a root of $B^{\prime}(s)=0$ on $[0, \infty)$ and thus $\xi_{S}=0$ and then $B^{\prime}(s)$ is strictly decreasing until its minimum point $\eta$ and thus we must have $B^{\prime}(s)<0$ for all $s \in(0, \eta]$. After hitting $\eta, B^{\prime}(s)$ is then strictly increasing on $(\eta,+\infty)$ and since $\lim _{s \rightarrow+\infty} B^{\prime}(s)=+\infty$, we know that $B^{\prime}(s)$ must have exactly one zero, denoted by $\xi_{L}$, on $(\eta,+\infty)$. It follows that $B(s)$ is strictly decreasing on $\left[0, \xi_{L}\right)$ (recall $\xi_{S}=0$ ) and strictly increasing on $\left(\xi_{L}, \infty\right)$ and thus $B(s)$ attains its minimum value $B\left(\xi_{L}\right)$ at $\xi_{L}$. Note that $B(0)=b_{0}>0, B(1)=-d \leq 0$ and $\lim _{s \rightarrow+\infty} B(s)=+\infty$, it is clear that $B(s)$ has exactly two positive zeros $q_{S}$ and $q_{L}$ and all the conclusions in this Lemma 2.1 regarding the case of $b_{1}=0$ follow.

On the other hand, if $B^{\prime}(0)=b_{1}>0$, then $B^{\prime}(s)$ is strictly decreasing on $[0, \eta)$ from $b_{1}$, which is positive, to its minimal value $B^{\prime}(\eta)$. Now, we claim that $B^{\prime}(\eta)<0$. Indeed, if $B^{\prime}(\eta) \geq 0$, then $B^{\prime}(s)$ would be positive for all $s \neq \eta$ and hence $B(s)$ would be strictly increasing on $[0, \infty)$ which contradicts with the fact that $B(0)=b_{0}>B(1)=-d$. Therefore, we have proven that $B^{\prime}(\eta)<0$. It then easily follows that $B^{\prime}(s)$ has exactly two zeros $\xi_{S}$ and $\xi_{L}$ such that $0<\xi_{S}<\eta<\xi_{L}$ and that $B^{\prime}(s)<0$ for $s \in\left(\xi_{S}, \xi_{L}\right)$ and that $B^{\prime}(s)>0$ for $s \in\left[0, \xi_{S}\right) \cup\left(\xi_{L}, \infty\right)$. Then $B(s)$ is strictly increasing on $\left[0, \xi_{S}\right] \cup\left[\xi_{L}, \infty\right)$ and strictly decreasing on $\left[\xi_{S}, \xi_{L}\right]$. Now, beginning from $B(0)=b_{0}>0, B(s)$ increases on $\left[0, \xi_{S}\right)$ until its local maximum $B\left(\xi_{S}\right)$, which must be positive, and then decreases on $\left[\xi_{S}, \xi_{L}\right]$, until its minimum value $B\left(\xi_{L}\right)$. Of course, we have $B\left(\xi_{L}\right) \leq 0$ (because of $B(1) \leq 0$ ) with $B^{\prime}\left(\xi_{L}\right)=0$ if and only if $B(1)=B^{\prime}(1)=0$. After reaching the non-positive minimum value $B\left(\xi_{L}\right)$, $B(s)$ then increases to $+\infty$ as $s \rightarrow+\infty$. Hence, $B(s)$ must have exactly two positive zeros $q_{S}$ and $q_{L}$ such that $0<\xi_{S}<q_{S}<\xi_{L}<q_{L}<\infty$ except that in the case of $B(1)=B^{\prime}(1)=0$ we have $0<\xi_{S}<q_{S}=\xi_{L}=q_{L}=1$. All other conclusions now easily follow.

Remark 2.1. By Lemma 2.1 and its proof, we also see that $B(s)$ is concave on $[0, \eta)$ and convex on $[\eta, \infty)$ with $s=\eta$ as the inflection point, where $\eta$ is the unique positive zero of $B^{\prime \prime}(s)$. This $\eta$, together with the other four important quantities $\xi_{S}, q_{S}, \xi_{L}$ and $q_{L}$ determines the local properties of $B(s)$. For example, we may easily derive the intervals on which $B(s)$ is negative, decreasing and concave, etc.

Lemma 2.1 completely describes the behavior of $B(s)$ for $\rho=+\infty$. If $\rho<+\infty$, then the principle of the above proof still works. The only difference is that, of course, we can only consider the behavior of $B(s)$ on the finite interval $[0, \rho)$ and that we may not have the property that $B^{\prime \prime \prime}(\rho)$, say, is infinity. Therefore, with some obvious amendments, we may easily prove the following conclusion.

Lemma 2.2. Assume that $\rho<+\infty$.

(i) If $B(\rho)>0$ ( including $B(\rho)=+\infty)$ or, if $B(\rho)=0$ but $\rho>1$, then all the conclusions of Lemma 2.1 hold. In particular, each of $B(s)$ and $B^{\prime}(s)$ has exactly two interlaced zeros $0 \leq \xi_{S}<q_{S}<\xi_{L}<q_{L}$.

(ii) If $B(\rho)<0$ and $B^{\prime}(\rho) \geq 0$ (including $B^{\prime}(\rho)=+\infty$ ), then $B^{\prime}(s)$ has two nonnegative zeros $0 \leq \xi_{S}<\xi_{L} \leq \rho$ with $\xi_{S}=0$ if and only if $b_{1}=0$ and $\xi_{L}=\rho$ if and only if $B^{\prime}(\rho)=0$ with $B^{\prime}(s)<0$ for $s \in\left(\xi_{S}, \xi_{L}\right)$ and $B^{\prime}(s)>0$ for $s \in\left(0, \xi_{S}\right) \cup\left(\xi_{L}, \rho\right)$. Moreover, $B(s)$ has exactly one nonnegative zero $q_{S} \in\left(\xi_{S}, \xi_{L}\right)$ with $B(s)>0$ for $s \in\left[0, q_{S}\right)$ and $B(s)<0$ for $s \in\left(q_{S}, \rho\right]$. 
(iii) If $B(\rho)<0$ and $B^{\prime}(\rho)<0$, then each of $B^{\prime}(s)$ and $B(s)$ has exactly one zero, $\xi_{S}$ and $q_{S}$, respectively, such that $0 \leq \xi_{S}<q_{S}<\rho$ with $\xi_{S}=0$ if and only if $b_{1}=0$. Moreover, $B^{\prime}(s)>0$ for $s \in\left(0, \xi_{S}\right)$ and $B^{\prime}(s)<0$ for $s \in\left(\xi_{S}, \rho\right]$ and $B(s)>0$ for $s \in\left[0, q_{S}\right)$ and $B(s)<0$ for $s \in\left(q_{S}, \rho\right]$.

Remark 2.2. If $B(\rho)=0, \rho=1$ and $B^{\prime}(\rho) \leq 0$ then $B(s)$ has exact one zero $q_{S}=1$ and $B^{\prime}(s)$ has a zero $\xi_{S} \in[0,1)$. Furthermore, $\rho=1$ is a zero of $B^{\prime}(s)$ if and only if $B^{\prime}(\rho)=0$. If $B(\rho)=0, \rho=1$ and $B^{\prime}(\rho)>0$ then $B(s)$ has exact two zeros $0<q_{S}<q_{L}=1$ and $B^{\prime}(s)$ has exact two zeros $\xi_{S}, \xi_{L}$ such that $0 \leq \xi_{S}<q_{S}<\xi_{L}<q_{L}=1$.

In connection with $B(s)$ and $B^{\prime}(s)$, we define a new function

$$
g(s)=s B^{\prime}(s)-2 B(s), \quad s \in(-\rho, \rho) .
$$

Obviously, $g(s)$ is also a power series with the same convergence radius $\rho$ as $B(s)$. We shall see later that the function $g(s)$ plays a role as the "testing function" in determining the decay parameter and hence it is crucial to understand the properties of $g(s)$.

Lemma 2.3. The function $g(s)$ is convex on $(0, \rho)$ and there are only two possibilities for the nonnegative zeros of $g(s)$. That is that either $g(s)$ has only one zero or no zero on $[0, \rho]$ (if $\rho=+\infty,[0, \rho]$ should be read as $[0, \rho)$ ). More specifically,

(i) If $\rho=+\infty$, then there exists a unique $s_{*} \in(0, \infty)$ such that $g\left(s_{*}\right)=0$ and that $g(s)<0$ for all $s \in\left(0, s_{*}\right)$ and $g(s)>0$ for all $s \in\left(s_{*}, \infty\right)$.

(ii) If $\rho<+\infty$ and $B(\rho)=+\infty$, then there exists a unique $s_{*} \in(0, \rho)$ such that $g\left(s_{*}\right)=0$ and that $g(s)<0$ for all $s \in\left(0, s_{*}\right)$ and $g(s)>0$ for all $s \in\left(s_{*}, \rho\right)$.

(iii) If $\rho<+\infty, B(\rho)<+\infty$ and $\sum_{j=3}^{\infty}(j-2) b_{j} \rho^{j} \geq b_{1} \rho+2 b_{0}$, then there exists $s_{*} \in(0, \rho]$ such that $g\left(s_{*}\right)=0$ and that $g(s)<0$ for all $s \in\left(0, s_{*}\right)$ and $g(s)>0$ for all $s \in\left(s_{*}, \rho\right]$. Furthermore, $s_{*}=\rho$ if and only if $\sum_{j=3}^{\infty}(j-2) b_{j} \rho^{j}=b_{1} \rho+2 b_{0}$.

(iv) If $\rho<+\infty$ and $\sum_{j=3}^{\infty}(j-2) b_{j} \rho^{j}<b_{1} \rho+2 b_{0}$, then $g(s)<0$ for all $s \in[0, \rho]$ and thus there exists no zero for $g(s)$ on $[0, \rho]$.

Moreover, for the first three cases we have $g^{\prime}\left(s_{*}\right)>0$.

Proof. Note that for $0 \leq s<\rho$, we have

$$
g^{\prime}(s)=s B^{\prime \prime}(s)-B^{\prime}(s)
$$

and

$$
g^{\prime \prime}(s)=s B^{\prime \prime \prime}(s)
$$

Hence, $g^{\prime \prime}(s)>0$ for all $s \in(0, \rho)$ due to the fact that $B^{\prime \prime \prime}(s)>0$ on the same interval. Therefore $g(s)$ is convex on $[0, \rho)$ and $g^{\prime}(s)$ is strictly increasing on $[0, \rho)$. It follows that $g(s)$ has at most two zeros on $[0, \rho)$ (or $[0, \rho]$ if $\rho<+\infty$ ). Now we further claim that $g(s)$ has at most one nonnegative zero. Indeed, since $g^{\prime}(s)$ is strictly increasing on $[0, \rho)$ and that $g^{\prime}(0)=-B^{\prime}(0)=-b_{1} \leq 0$, we know that if $b_{1}=0$ then $g^{\prime}(0)=0$ and thus $g^{\prime}(s)>0$ for all $s \in(0, \rho)$ since $g^{\prime}(s)$ is strictly increasing. This implies that $g(s)$ is strictly increasing on $(0, \rho)$ and thus $g(s)$ has at most one zero on $[0, \rho)$. Furthermore, since $g(0)=-2 B(0)=-2 b_{0}<0$ we see that $g(s)$ changes its sign from negative to positive in crossing this unique nonnegative zero. On the other hand, if $b_{1}>0$ then $g^{\prime}(0)<0$. 
Considering $g^{\prime}(s)$ strictly increases on $[0, \rho)$, we know that there are only two possibilities for signs of $g^{\prime}(s)$. That is that either $g^{\prime}(s)<0$ for all $s \in(0, \rho)$ or $g^{\prime}(s)$ changes its signs from negative into positive in some point (which is the zero of $g^{\prime}(s)$ ) in $(0, \rho]$. It follows that either $g(s)$ decreases on $(0, \rho)$ or $g(s)$ changes its decreasing property into increasing property at the zero of $g^{\prime}(s)$. Now $g(0)=-2 b_{0}<0$ and thus either $g(s) \leq 0$ for all $s \in(0, \rho)$ (if $\rho<+\infty,(0, \rho)$ should be read as $(0, \rho])$ or $g(s)$ could become positive in some point. It is clear that the former can happen if and only if $\rho<+\infty$ and $g(\rho) \leq 0$, or equivalently, if and only if $\rho<+\infty$ and $\sum_{j=3}^{\infty}(j-2) b_{j} \rho^{j} \leq 2 b_{0}+b_{1} \rho$. The last equivalency is due to the fact that

$$
g(s)=\sum_{j=3}^{\infty}(j-2) b_{j} s^{j}-2 b_{0}-b_{1} s .
$$

Now all the conclusions except the last part in the lemma easily follow.

We now prove the last part, i.e., $g\left(s_{*}\right)=0$ implies $g^{\prime}\left(s_{*}\right)>0$. However, this is easy. Indeed, we know that $g\left(s_{*}\right)=0$ is just

$$
\sum_{j=3}^{\infty}(j-2) b_{j} s_{*}^{j}=b_{1} s_{*}+2 b_{0}
$$

or

$$
\sum_{j=3}^{\infty}(j-2) b_{j} s_{*}^{j-1}-\frac{2 b_{0}}{s_{*}}=b_{1}
$$

since $s_{*}>0$. Now, by $(2.6)$ we have

$$
g^{\prime}\left(s_{*}\right)=\sum_{j=3}^{\infty} j(j-2) b_{j} s_{*}^{j-1}-b_{1}
$$

Substituting (2.8) into (2.9) yields that

$$
g^{\prime}\left(s_{*}\right)=\sum_{j=3}^{\infty}(j-1)(j-2) b_{j} s_{*}^{j-1}+\frac{2 b_{0}}{s_{*}}
$$

which is obviously positive.

Lemma 2.4. Let $Q$ and $\tilde{Q}$ be two generator matrices as defined in Definition 1.1 (neither is assumed to be necessarily conservative) which are determined by two sequences $\left\{b_{n} ; n \geq 0\right\}$ and $\left\{\tilde{b}_{n} ; n \geq 0\right\}$. Suppose that

$$
\tilde{b}_{n}=a^{n} b_{n}, \quad n \geq 0 \text { where } a>0 .
$$

Then $Q$ and $\tilde{Q}$ as well as their corresponding transition functions have a common irreducible class $C=\{2,3, \cdots\}$. Moreover, their corresponding decay parameters, denoted by $\lambda_{C}$ and $\tilde{\lambda}_{C}$, respectively, have the relationship

$$
\tilde{\lambda}_{C}=a^{2} \lambda_{C}
$$


Proof. It is obvious that $Q$ and $\tilde{Q}$ have the same irreducible class $C$ and hence we only need to prove (2.11). We first show that

$$
\tilde{\lambda}_{C} \geq a^{2} \lambda_{C}
$$

Note that $\lambda_{C}$ is the decay parameter for $Q$ on $C$, we know that there exists a $\lambda_{C}$-subinvariant vector $\left(x_{j} ; j \geq 2\right)$ such that $x_{j}>0(j \geq 2)$ and

$$
\left\{\begin{array}{l}
\sum_{j=2}^{\infty} b_{j} x_{j} \leq-\lambda_{C} x_{2} \\
\sum_{j=1}^{\infty} b_{j} x_{j+1} \leq-\lambda_{C} x_{3} \\
\sum_{j=0}^{\infty} b_{j} x_{j+i-2} \leq-\lambda_{C} x_{i}, \quad i \geq 4 .
\end{array}\right.
$$

Now, let $\tilde{x}_{j}=x_{j} / a^{j}(j \geq 2)$. It is obvious that $\tilde{x}_{j}>0(j \geq 2)$ and that we have for $i=2$,

$$
\sum_{j=2}^{\infty} \tilde{b}_{j} \tilde{x}_{j}=\sum_{j=2}^{\infty} b_{j} x_{j} \leq-\lambda_{C} x_{2}=-\left(a^{2} \lambda_{C}\right) \tilde{x}_{2}
$$

and for $i=3$,

$$
\sum_{j=1}^{\infty} \tilde{b}_{j} \tilde{x}_{j+1}=\frac{1}{a} \cdot \sum_{j=1}^{\infty} b_{j} x_{j+1} \leq-\lambda_{C} x_{3} / a=-\left(a^{2} \lambda_{C}\right) \tilde{x}_{3},
$$

and finally for $i \geq 4$

$$
\sum_{j=0}^{\infty} \tilde{b}_{j} \tilde{x}_{j+i-2}=\frac{1}{a^{i-2}} \cdot \sum_{j=0}^{\infty} b_{j} x_{j+i-2} \leq-\lambda_{C} x_{i} / a^{i-2}=-\left(a^{2} \lambda_{C}\right) \tilde{x}_{i} .
$$

Therefore, $\left(\tilde{x}_{j}, j \geq 2\right)$ is a $a^{2} \lambda_{C}$-subinvariant vector for $\tilde{Q}$ on $C$ and hence (2.12) is proved.

However, noting $a>0$, we may get from (2.10) that $b_{n}=\tilde{b}_{n} / a^{n}(n \geq 0)$ and thus by the same argument as above we may obtain

$$
\lambda_{C} \geq \tilde{\lambda}_{C} / a^{2} .
$$

By (2.12) and (2.17), we know that (2.11) is true.

Lemma 2.5. Suppose that $Q$ and $\tilde{Q}$ are two generator matrices defined on the same state space $\mathbf{E}$ satisfying

$$
\tilde{Q}=Q+\alpha I
$$

where $I$ is the identity matrix on $\mathbf{E} \times \mathbf{E}$ and $\alpha$ is a real number. Then the corresponding Feller $Q$ - and $\tilde{Q}$-functions, denoted by $P(t)=\left(p_{i j}(t) ; i, j \in \mathbf{E}\right)$ and $\tilde{P}(t)=\left(\tilde{p}_{i j}(t) ; i, j \in \mathbf{E}\right)$ have the relationship

$$
\tilde{p}_{i j}(t)=e^{\alpha t} p_{i j}(t), \quad i, j \in \mathbf{E}, t \geq 0 .
$$

In particular, if $\tilde{P}(t)$ and $P(t)$ have the same irreducible class $C$, then

$$
\tilde{\lambda}_{C}=\lambda_{C}-\alpha
$$

where $\lambda_{C}$ and $\tilde{\lambda}_{C}$ are decay parameters for $P(t)$ and for $\tilde{P}(t)$ on $C$, respectively. 
Proof. (2.19) is trivial and then (2.20) follows from (2.19) and (1.1).

Note that Lemma 2.5 holds true for any two generator matrices, not necessarily for the BABS generator matrices. Now in combining Lemmas 2.4 and 2.5, we may get the following useful lemma which will be one of our basic methods in determining the decay parameter and the invariant measures for the stopped BABS queues.

Lemma 2.6. Suppose that $Q$ is a $B A B S$ generator matrix as defined in Definition 1.1 and $C=\{2,3, \cdots\}$ is an irreducible class for $Q$. Define a new sequence $\left\{\tilde{b}_{n} ; n \geq 0\right\}$ as

$$
\tilde{b}_{n}=b_{n} \beta^{n}(n \neq 2) \text { and } \tilde{b}_{2}=b_{2} \beta^{2}+\alpha \beta^{2}
$$

where $\beta>0$ and $\alpha \geq 0$. Let $\tilde{Q}=\left(\tilde{q}_{i j} ; i, j \geq 0\right)$ be defined as

$$
\tilde{q}_{i j}= \begin{cases}\tilde{b}_{j-i+2}, & \text { if } i \geq 2, j \geq i-2 \\ 0, & \text { otherwise }\end{cases}
$$

Assume that

$$
\sum_{n=0}^{\infty} \tilde{b}_{n} \leq 0
$$

Then $\tilde{Q}$ is also a BABS generator matrix as defined in Definition 1.1 and $\tilde{Q}$ is conservative if and only if the equality in (2.23) holds. Furthermore, $C=\{2,3, \cdots\}$ is a common irreducible class for $Q$ and $\tilde{Q}$ and we have

$$
\tilde{\lambda}_{C}=\beta^{2} \lambda_{C}-\alpha \beta^{2}
$$

where $\tilde{\lambda}_{C}$ and $\lambda_{C}$ are the decay parameter of $\tilde{Q}$-function $\tilde{P}(t)$ and $Q$-function $P(t)$, respectively.

Proof. By $(2.21)$, it is clear that $\tilde{b}_{n} \geq 0(n \neq 2)$. Condition $(2.23)$ then guarantees that $\tilde{b}_{2}<0$ and that $\tilde{Q}$ given in $(2.22)$ is indeed a BABS generator matrix. Furthermore, since $\tilde{b}_{n}>0(n \neq 2)$ if and only if $b_{n}>0(n \neq 2)$ and thus $\tilde{Q}$ also satisfies all the additional requirements $(1.4)-(1.5)$. Hence $C=\{2,3, \cdots\}$ is a common irreducible class for both $Q$ and $\tilde{Q}$. Now the conclusion (2.24) follows from Lemmas 2.4 and 2.5 .

Remark 2.3. By Lemma 2.6, we see that the essential condition is (2.23). Also note that both states 0 and 1 are common absorbing states for $Q$ and for $\tilde{Q}$. In other words, in applying Lemma 2.5, when defining $\tilde{Q}=Q+\alpha I$ as in (2.18), the $\alpha I$ is actually confined on $C=\{2,3, \cdots\}$ only.

\section{Decay parameter}

We are now ready to determine the decay parameter $\lambda_{C}$ for our stopped BABS queueing processes, where $C=\{2,3, \cdots\}$. Similarly as in Li and Chen [27], define

$$
\rho_{0}=\sup \{s \geq 0 ; B(s) \leq 0\}
$$


and

$$
\begin{aligned}
\lambda_{*} & =\sup \left\{\lambda \geq 0 ; B(s)+\lambda s^{2}=0 \text { has a root in }\left[0, \rho_{0}\right]\right\} \\
& =\sup \left\{\lambda \geq 0 ; B(s)+\lambda s^{2}=0 \text { has a root in }\left[q_{S}, \rho_{0}\right]\right\} .
\end{aligned}
$$

By Lemmas 2.1 and 2.2 , it is easily seen that both $\rho_{0}$ and $B\left(\rho_{0}\right)$ are finite and that there are only two possibilities for $\rho_{0}$ : either $\rho_{0}=q_{L}$ or $\rho_{0}=\rho<+\infty$. Indeed, for all cases in Lemma 2.1 and the first case in Lemma 2.2 we have $\rho_{0}=q_{L}$ while for the last two cases in Lemma 2.2 we have $\rho_{0}=\rho<+\infty$. The last equality in (3.2) also easily follows from Lemmas 2.1 and 2.2 .

We shall see later that the supremum in (3.2) is attainable and, more importantly, is just the decay parameter for our stopped BABS queue. Hence, it is very informative and useful to give further characteristics of this important quantity, including its geometric meaning. To achieve this aim, we define

$$
\bar{\lambda}=\max \left\{-\frac{B(s)}{s^{2}} ; s \in\left[q_{S}, \rho_{0}\right]\right\}=\max \left\{-\frac{B(s)}{s^{2}} ; s \in\left[0, \rho_{0}\right]\right\} .
$$

Of course, this quantity can also be expressed as

$$
\bar{\lambda}=-\min \left\{\frac{B(s)}{s^{2}} ; s \in\left[q_{S}, \rho_{0}\right]\right\}=-\min \left\{\frac{B(s)}{s^{2}} ; s \in\left[0, \rho_{0}\right]\right\} .
$$

Since we have assumed that $b_{0}>0$ which implies $q_{S}>0$, we know that $-\frac{B(s)}{s^{2}}$ is continuous on $\left[q_{S}, \rho_{0}\right]$. Therefore, (3.3) is well-defined. That is that there exists $\bar{s} \in\left[q_{S}, \rho_{0}\right]$ such that $\bar{\lambda}=-\frac{B(\bar{s})}{\bar{s}^{2}}$. Note, again, that the last equality in both (3.3) and (3.4) easily follows from Lemmas 2.1 and 2.2.

Lemma 3.1. $\lambda_{*}=\bar{\lambda}$.

Proof. Firstly, it is clear that $\bar{\lambda} \leq \lambda_{*}$. Indeed, by (3.3) we know that $B(\bar{s})+\bar{\lambda} \bar{s}^{2}=0$ where $\bar{s} \in\left[q_{S}, \rho_{0}\right]$ and thus by (3.2) we obtain $\bar{\lambda} \leq \lambda_{*}$. In order to prove the converse, we assume that $\bar{\lambda}<\lambda_{*}$. It follows that there exists a $\mu \in\left(\bar{\lambda}, \lambda_{*}\right)$. Since $\mu<\lambda_{*}$, we know that the equation $B(s)+\mu s^{2}=0$ has a root $s_{\mu} \in\left[q_{S}, \rho_{0}\right]$ which just means that $\mu=-\frac{B\left(s_{\mu}\right)}{s_{\mu}^{2}}$. Hence by (3.3) we have $\mu \leq \bar{\lambda}$ which is a contradiction.

Lemma 3.2. (i) For the three cases (i)-(iii) in Lemma 2.3, we have

$$
\lambda_{*}=-\frac{B\left(s_{*}\right)}{s_{*}^{2}}=-\frac{B^{\prime}\left(s_{*}\right)}{2 s_{*}}
$$

where $s_{*}$ is the unique root of the equation $B(s)+\lambda_{*} s^{2}=0$ in $\left[0, \rho_{0}\right]$ and, also, is the same $s_{*}$ as defined in Lemma 2.3.

(ii) For case (iv) in Lemma 2.3, we have

$$
\lambda_{*}=-\frac{B\left(\rho_{0}\right)}{\rho_{0}^{2}} .
$$


Proof. Let $f(s)=\frac{B(s)}{s^{2}}\left(s \in\left(0, \rho_{0}\right]\right)$, then

$$
f^{\prime}(s)=\frac{s B^{\prime}(s)-2 B(s)}{s^{3}}=\frac{g(s)}{s^{3}}
$$

where $g(s)$ is defined in (2.5). It follows from (3.5) that

$$
f^{\prime \prime}(s)=\frac{g^{\prime}(s)}{s^{3}}-\frac{3 g(s)}{s^{4}} .
$$

By (3.5), it is clear that there exists a $s_{*} \in\left[q_{S}, \rho_{0}\right]$ such that $f^{\prime}\left(s_{*}\right)=0$ if and only if $g\left(s_{*}\right)=0$. For this $s_{*}$, we can get from (3.6) and Lemma 2.3 that

$$
f^{\prime \prime}\left(s_{*}\right)=\frac{g^{\prime}\left(s_{*}\right)}{s_{*}^{3}}-\frac{3 g\left(s_{*}\right)}{s_{*}^{4}}=\frac{g^{\prime}\left(s_{*}\right)}{s_{*}^{3}}>0 .
$$

Hence the function $f(s)$ attains its minimum value at $s=s_{*}$. Conclusion (i) thus follows. To prove (ii), just note that for this case we have $g(s)<0$ for all $s \in\left(0, \rho_{0}\right]$ and thus by (3.5) we also have $f^{\prime}(s)<0$ for all $s \in\left(0, \rho_{0}\right]$. It follows that $f(s)$ is strictly decreasing on $\left(0, \rho_{0}\right]$ and thus attains its minimum at point $\rho_{0}$.

Lemma 3.3. Let $g(s)$ be given in (2.5). Then $g\left(\rho_{0}\right) \geq 0$ if and only if one of the following situations occurs.

(i) $\rho=\infty$.

(ii) $\rho<\infty$ and $0 \leq B(\rho) \leq+\infty$.

(iii) $\rho<\infty, B(\rho)<0$ and $B^{\prime}(\rho) \geq 0$.

(iv) $\rho<\infty, B(\rho)<0$ and $B^{\prime}(\rho)<0$ and $\rho B^{\prime}(\rho) \geq 2 B(\rho)$.

Thus $g\left(\rho_{0}\right)<0$ if and only if $\rho<\infty, B(\rho)<0, B^{\prime}(\rho)<0$ and $\rho B^{\prime}(\rho)<2 B(\rho)$.

Proof. In cases (i) and (ii), we have $\rho_{0}=q_{L}$ and thus $B\left(q_{L}\right)=0$. However, by Lemmas 2.1 and 2.2 we know that $B^{\prime}\left(q_{L}\right)>0$ and thus $g\left(\rho_{0}\right)>0$. For case (iii), $\rho_{0}=\rho$ and thus since $B(\rho)<0$ and $B^{\prime}(\rho) \geq 0$ we know that $g\left(\rho_{0}\right)>0$. Case (iv) is just the condition that $g\left(\rho_{0}\right) \geq 0$ for all other cases. The proof is complete.

We now present our main result in this section by specifying the decay parameter. We shall present this result in two theorems dealing with two different cases.

Theorem 3.1. Suppose that $Q$ is a stopped BABS generator matrix as defined in Definition 1.1. If $\rho_{0} B^{\prime}\left(\rho_{0}\right) \geq 2 B\left(\rho_{0}\right)$ (that is $g\left(\rho_{0}\right) \geq 0$ ), then the decay parameter $\lambda_{C}$ for $Q$ on $C=\{2,3, \cdots\}$ is

$$
\lambda_{C}=\lambda_{*}
$$

Proof. We first consider the very special case of $B(1)=B^{\prime}(1)=0$ (that is $d=0$ and $\left.m_{d}=m_{b}\right)$. For this case, it is trivial to see that $\lambda_{*}=0$. We now claim that for this case we also have $\lambda_{C}=0$. Assume, on the contrary that $\lambda_{C}>0$. Then for any $\lambda \in\left(0, \lambda_{C}\right)$, we have $\int_{0}^{\infty} e^{\lambda t} p_{i j}(t) d t<+\infty$ for all $i, j \geq 2$, where $\left(p_{i j}(t) ; i, j \geq 0\right)$ is the $Q$-function. However, by the Kolmogorov forward equation we know that, for example,

$$
p_{20}^{\prime}(t)=b_{0} p_{22}(t) \quad \text { and } \quad p_{21}^{\prime}(t)=b_{1} p_{22}(t)+b_{0} p_{23}(t)
$$


and hence

$$
\int_{0}^{\infty} e^{\lambda t}\left(p_{20}^{\prime}(t)+p_{21}^{\prime}(t)\right) d t<+\infty
$$

Since $\lambda>0$ and using the inequality $e^{\lambda t} \geq 1+\lambda t$, we can obtain by applying (3.9) that

$$
\int_{0}^{\infty} t\left(p_{20}^{\prime}(t)+p_{21}^{\prime}(t)\right) d t<+\infty
$$

Note that (3.10) is just saying that $E_{2}[\tau]<+\infty$ where $\tau$ is the overall extinction time and $E_{2}[\cdot]$ is the conditional expectation when the process starts at state 2 (see Chen et al [8]). This contradicts Theorem 2 in Chen et al [8]. Therefore, (3.8) is true if $B(1)=B^{\prime}(1)=0$.

Now, we consider the general case under the condition $\rho_{0} B^{\prime}\left(\rho_{0}\right) \geq 2 B\left(\rho_{0}\right)$. By Lemma 3.2 we know that there exists a $s_{*} \in\left(0, \rho_{0}\right]$ such that

$$
B\left(s_{*}\right)=-\lambda_{*} s_{*}^{2} \quad \text { and } \quad B^{\prime}\left(s_{*}\right)=-2 \lambda_{*} s_{*} .
$$

Now, define

$$
\tilde{b}_{k}=b_{k} s_{*}^{k}, \quad k \neq 2
$$

and

$$
\tilde{b}_{2}=b_{2} s_{*}^{2}+\lambda_{*} s_{*}^{2} .
$$

It is easily seen that $\tilde{b}_{k} \geq 0(k \neq 2)$ and

$$
\sum_{k=0}^{\infty} \tilde{b}_{k}=B\left(s_{*}\right)+\lambda_{*} s_{*}^{2}=0 .
$$

Hence, (2.23) is satisfied. Therefore, if we define $\tilde{Q}$ as in (2.22), then $\tilde{Q}$ is a conservative stopped BABS generator matrix as defined in Definition 1.1 and then by Lemma 2.6, we have

$$
\tilde{\lambda_{C}}=\lambda_{C} s_{*}^{2}-\lambda_{*} s_{*}^{2}
$$

For this $\tilde{Q}$, we now define

$$
\tilde{B}(s)=\sum_{j=0}^{\infty} \tilde{b}_{j} s^{j} .
$$

Then it is easy to see that $\tilde{B}(1)=\tilde{B}^{\prime}(1)=0$. Therefore, by the proven (3.8) for this special case, we know that

$$
\tilde{\lambda}_{C}=0
$$

which, together with (3.14), immediately yields that $\lambda_{C}=\lambda_{*}$ since $s_{*}>0$.

In order to consider the case of $\rho_{0} B^{\prime}\left(\rho_{0}\right)<2 B\left(\rho_{0}\right)$, we provide another lemma. 
Lemma 3.4. Suppose that the given stopped BABS generator matrix $Q$ is conservative and let $B(s)$ be the generating function given by (2.1). Assume that $\rho=1$ and $B^{\prime}(1)<0$, then $\lambda_{C}=\lambda_{*}=0$.

Proof. First, considering that $B(s)$ is not defined for $s>1$ due to the assumption that $\rho=1$, we define $B(s)=\infty$ for all $s>1$ for technical reasons. Now since $B(1)=0$, $B^{\prime}(1)<0$ and $\rho=1$, it is clear that $\lambda_{*}=0$. Hence we only need to prove $\lambda_{C}=0$. For this purpose, choose $N_{0} \geq 3$ such that $b_{N_{0}}>0$ and for any $n \geq N_{0}$, we define

$$
b_{k}^{(n)}= \begin{cases}b_{k}, & \text { if } k \leq n \\ 0, & \text { if } k>n\end{cases}
$$

and then define a generator matrix $Q^{(n)}=\left(q_{i j}^{(n)} ; i, j \geq 0\right)$ as

$$
q_{i j}^{(n)}= \begin{cases}b_{j-i+2}^{(n)}, & \text { if } i \geq 2, j \geq i-2 \\ 0, & \text { otherwise }\end{cases}
$$

It is clear that $Q^{(n)}$ is a nonconservative stopped BABS generator matrix and $C$ is an irreducible class for each $Q^{(n)}\left(n \geq N_{0}\right)$. Let

$$
B_{n}(s)=\sum_{k=0}^{\infty} b_{k}^{(n)} s^{k}
$$

and

$$
\lambda_{*}^{(n)}=\sup \left\{\lambda \geq 0 ; B_{n}(s)+\lambda s^{2}=0 \text { has a root in }\left[0, \rho_{0}^{(n)}\right]\right\}
$$

where $\rho_{0}^{(n)}=\sup \left\{s>0 ; B_{n}(s) \leq 0\right\}$. It is obvious that the convergence radius for each $B_{n}(s)$ is infinite and thus $\rho_{0}^{(n)} B_{n}^{\prime}\left(\rho_{0}^{(n)}\right)>2 B_{n}\left(\rho_{0}^{(n)}\right)$ (indeed, $B_{n}\left(\rho^{(n)}\right)=0$ and $\left.B_{n}^{\prime}\left(\rho^{(n)}\right)>0\right)$. It follows from Lemma 3.3 and Theorem 3.1 that the decay parameter for $Q^{(n)}$ is

$$
\lambda_{C}^{(n)}=\lambda_{*}^{(n)} .
$$

We also know that for each $n \geq N_{0}$, there exists $s_{*}^{(n)} \in\left[q_{S}^{(n)}, q_{L}^{(n)}\right]$ such that $\lambda_{C}^{(n)}=$ $\max \left\{-\frac{B_{n}(s)}{s^{2}} ; s \in\left[q_{S}^{(n)}, q_{L}^{(n)}\right]\right\}$, where $q_{S}^{(n)}$ and $q_{L}^{(n)}$ are the two zeros of $B_{n}(s)$ satisfying

$$
0<q_{S}^{(n)}<1<q_{L}^{(n)}<+\infty
$$

since $B_{n}(1)<0$ for each $n \geq N_{0}$.

By (3.15) and (3.16), it is easily seen that for all $s>0$,

$$
B_{n}(s) \leq B_{n+1}(s)<B(s)
$$

with $B_{n}(0)=B(0)\left(n \geq N_{0}\right)$. Intuitively speaking, (3.21) means that the whole curve $B_{n+1}(s)$ is above $B_{n}(s)$ and all $B_{n}(s)$ is below $B(s)$ for all $s \geq 0$. It follows that $q_{S}^{(n)}$ and $q_{L}^{(n)}$ are increasing and decreasing functions of $s$, respectively and that

$$
\left[q_{S}^{(n)}, q_{L}^{(n)}\right] \supset\left[q_{S}^{(n+1)}, q_{L}^{(n+1)}\right] .
$$


Therefore, both the limits $\lim _{n \rightarrow \infty} q_{S}^{(n)}$ and $\lim _{n \rightarrow \infty} q_{L}^{(n)}$ exist, denoted by $q_{S}^{(\infty)}$ and $q_{L}^{(\infty)}$ respectively. By (3.20),

$$
q_{S}^{(\infty)} \leq 1 \leq q_{L}^{(\infty)}
$$

Now, we claim that

$$
q_{S}^{(\infty)}=q_{L}^{(\infty)}=1 .
$$

Recall we already have that $q_{L}^{(\infty)} \geq 1$ and thus in order to prove $q_{L}^{(\infty)}=1$, it is sufficient to show that $B\left(q_{L}^{(\infty)}\right)<+\infty$ since $\rho=1$. However, we have that $B_{n}\left(q_{L}^{(n)}\right)=0$ for all $n$ which means that

$$
\sum_{k \neq 2} b_{k}^{(n)}\left(q_{L}^{(n)}\right)^{k}=-b_{2}\left(q_{L}^{(n)}\right)^{2}
$$

Since $q_{L}^{(n)} \Downarrow q_{L}^{(\infty)}$ as $n \rightarrow \infty$ and the left hand side of (3.23) is a nonnegative series, we conclude that for all $n$

$$
\sum_{k \neq 2} b_{k}^{(n)}\left(q_{L}^{(\infty)}\right)^{k} \leq-b_{2}\left(q_{L}^{(n)}\right)^{2}
$$

Therefore,

$$
\sum_{k \neq 2} b_{k}\left(q_{L}^{(\infty)}\right)^{k} \leq-b_{2}\left(q_{L}^{(\infty)}\right)^{2}<+\infty
$$

Hence $q_{L}^{(\infty)} \leq 1$ and thus $q_{L}^{(\infty)}=1$.

We now prove that $q_{S}^{(\infty)}=1$. Recall that $B_{n}\left(q_{S}^{(n)}\right)=0$, i.e.,

$$
\sum_{k \neq 2} b_{k}^{(n)}\left(q_{S}^{(n)}\right)^{k}=-b_{2}\left(q_{S}^{(n)}\right)^{2} .
$$

Now, each term in the left hand side of (3.25) is increasing with $n$ since both $b_{k}^{(n)}$ and $q_{S}^{(n)}$ are increasing and thus we may apply the monotone convergence theorem directly in (3.25) to obtain

$$
\sum_{k \neq 2} b_{k}\left(q_{S}^{(\infty)}\right)^{k}=-b_{2}\left(q_{S}^{(\infty)}\right)^{2}
$$

or $B\left(q_{S}^{(\infty)}\right)=0$. Since $B(1)=0$, we know that $q_{S}^{(\infty)}=1$. Therefore, we have proved (3.22). However, recall that $s_{*}^{(n)} \in\left(q_{S}^{(n)}, q_{L}^{(n)}\right)$ and it follows from (3.22) that we also have $\lim _{n \rightarrow \infty} s_{*}^{(n)}=1$.

Finally, let $\left.{ }_{(m)} P(t)={ }_{\left({ }_{(m)}\right.} p_{i j}(t) ; i, j \geq 0\right)$ and $P(t)=\left(p_{i j}(t) ; i, j \geq 0\right)$ be the Feller minimal $Q^{(m)}$-function and $Q$-function, respectively. It is well-known that (see p.71 in Anderson [1], for example) each ${ }_{(m)} P(t)\left(m \geq N_{0}\right)$ and $P(t)$ can be obtained by using the backward integral recursions

$$
{ }_{(m)} p_{i j}^{(n)}(t)= \begin{cases}\delta_{i j} e^{-q_{i} t}, & \text { if } n=0 \\ { }_{(m)} p_{i j}^{(0)}(t)+\int_{0}^{t} e^{-q_{i} s} \sum_{k \neq i} q_{i k}^{(m)}{ }_{(m)} p_{k j}^{(n-1)}(t-s) d s, & \text { if } n \geq 1\end{cases}
$$




$$
p_{i j}^{(n)}(t)= \begin{cases}\delta_{i j} e^{-q_{i} t}, & \text { if } n=0 \\ p_{i j}^{(0)}(t)+\int_{0}^{t} e^{-q_{i} s} \sum_{k \neq i} q_{i k} p_{k j}^{(n-1)}(t-s) d s, & \text { if } n \geq 1\end{cases}
$$

respectively, where $\delta_{i j}$ is the Kronecker delta, i.e.,

$$
\delta_{i j}= \begin{cases}1, & \text { if } i=j \\ 0, & \text { if } i \neq j\end{cases}
$$

Therefore, ${ }_{(m)} p_{i j}^{(n)}(t) \uparrow_{(m)} p_{i j}(t)$ and $p_{i j}^{(n)}(t) \uparrow p_{i j}(t)$ as $n \rightarrow \infty$. By (3.26), (3.27), (3.17) and (3.18), it is easily seen that for all $n \geq 0$,

$$
{ }_{(m)} p_{i j}^{(n)}(t) \leq_{(m+1)} p_{i j}^{(n)}(t) \leq p_{i j}^{(n)}(t), \quad \text { for all } i, j \geq 0 \text { and } t \geq 0
$$

and thus

$$
{ }_{(m)} p_{i j}(t) \leq_{(m+1)} p_{i j}(t) \leq p_{i j}(t), \quad \text { for all } i, j \geq 0 \text { and } t \geq 0 .
$$

It follows from (1.1) and (3.28) that

$$
\lambda_{C}^{(m)} \geq \lambda_{C}^{(m+1)} \geq \lambda_{C}
$$

That is that $\lambda_{C}^{(m)}$ is decreasing with $m$ and thus the $\operatorname{limit} \lim _{m \rightarrow \infty} \lambda_{C}^{(m)}$ exists, denoted by $\lambda_{C}^{(\infty)}$, and that

$$
\lambda_{C}^{(\infty)} \geq \lambda_{C}
$$

But we have $\lambda_{C}^{(n)}=\lambda_{*}^{(n)}=-\frac{B_{n}\left(s_{*}^{(n)}\right)}{\left(s_{*}^{(n)}\right)^{2}}$. Noting $\lim _{n \rightarrow \infty} s_{*}^{(n)}=1$ and using a similar technique as above yields that

$$
\lim _{n \rightarrow \infty} \lambda_{C}^{(n)}=-B(1)=0
$$

Hence, by (3.30) we obtain $\lambda_{C}=0$ which completes the proof.

Now we can present the final result regarding the decay parameter.

Theorem 3.2. Suppose that $Q$ is a stopped BABS generator matrix as defined in (1.2)(1.3), determined by the sequence $\left\{b_{k} ; k \geq 0\right\}$. Then the decay parameter $\lambda_{C}=\lambda_{*}$ where $\lambda_{*}$ is given by either (i) or (ii) in Lemma 3.2.

Proof. By Theorem 3.1, we now only need to consider the situation $B\left(\rho_{0}\right)>\frac{\rho_{0} B^{\prime}\left(\rho_{0}\right)}{2}$. However, for this case we know that $s_{*}=\rho$ and $\lambda_{*}=-\frac{B(\rho)}{\rho^{2}}$. Now, as a similar manner to the proof of Theorem 3.1, just define another sequence $\left\{\tilde{b}_{k} ; k \geq 0\right\}$ by letting $\tilde{b}_{k}=b_{k} \rho^{k}(k \neq 2)$, $\tilde{b}_{2}=b_{2} \rho^{2}+\lambda_{*} \rho^{2}$ and $\tilde{B}(s)=\sum_{k=0}^{\infty} \tilde{b}_{k} s^{k}$. It is then easy to see that $\tilde{B}(1)=0$ and $\tilde{B}^{\prime}(1)<0$, and thus by Lemma 3.4 we get $\tilde{\lambda}_{C}=0$. But by Lemma 2.6, again, we know that

$$
\tilde{\lambda}_{C}=\lambda_{C} \rho^{2}-\lambda_{*} \rho^{2}
$$

and thus $\lambda_{C}=\lambda_{*}$. 
The following conclusion reveals the clear geometric interpretation of our basic conclusion.

Theorem 3.3. (i) If $\rho_{0} B^{\prime}\left(\rho_{0}\right) \geq 2 B\left(\rho_{0}\right)$, then the two curves $y=B(s)$ and $y=-\lambda s^{2}$ contact with degree one at point $\left(s_{*}, B\left(s_{*}\right)\right)$. In other words, $-2 \lambda_{C} s_{*}$ is the slope of the common tangent line of the two curves $y=B(s)$ and $y=-\lambda s^{2}$ contact at a point whose abscissa is $s_{*}$.

(ii) If $\rho_{0} B^{\prime}\left(\rho_{0}\right)<2 B\left(\rho_{0}\right)$, then the two curves $y=B(s)$ and $y=-\lambda s^{2}$ contact with degree 0 at point $\left(s_{*}, B\left(s_{*}\right)\right)$.

Proof. Just note that two curves $y=f(x)$ and $y=g(x)$ contact with degree one at point $M$ with abscissa $x_{0}$ if and only if $f\left(x_{0}\right)=g\left(x_{0}\right), f^{\prime}\left(x_{0}\right)=g^{\prime}\left(x_{0}\right)$ and $f^{\prime \prime}\left(x_{0}\right) \neq g^{\prime \prime}\left(x_{0}\right)$ and contact with degree 0 at point $M^{\prime}$ with abscissa $x_{0}$ if and only if $f\left(x_{0}\right)=g\left(x_{0}\right)$ and $f^{\prime}\left(x_{0}\right) \neq g^{\prime}\left(x_{0}\right)$. Now all the conclusions easily follows from Lemma 3.2, Theorems 3.1 and 3.2 .

The following theorem gives the simple and practical calculation method for obtaining the decay parameter of the stopped BABS queue.

Theorem 3.4. Let $\rho_{0}$ be determined by (3.1) and $g(s)=s B^{\prime}(s)-2 B(s)$ be given by (2.5).

(i) If $g\left(\rho_{0}\right) \geq 0$, then $g(s)=0$ has a unique root $s_{*} \in\left[0, \rho_{0}\right]$ and thus $\lambda_{C}=-\frac{B^{\prime}\left(s_{*}\right)}{2 s_{*}}$. In other words, $s_{*}$ and $\lambda_{C}$ satisfy the equation

$$
\left\{\begin{array}{l}
s_{*} B^{\prime}\left(s_{*}\right)-2 B\left(s_{*}\right)=0 \\
\lambda_{C}=-\frac{B^{\prime}\left(s_{*}\right)}{2 s_{*}}
\end{array}\right.
$$

or equivalently, $s_{*}$ and $\lambda_{C}$ are the unique solution of the equation

$$
\left\{\begin{array}{l}
s B^{\prime}(s)-2 B(s)=0 \\
2 \lambda s+B^{\prime}(s)=0
\end{array}\right.
$$

in the unknowns $s$ and $\lambda$. Moreover, $s_{*}=\rho_{0}$ if and only if $g\left(\rho_{0}\right)=0$.

(ii) If $g\left(\rho_{0}\right)<0$, then $\lambda_{C}=-\frac{B\left(\rho_{0}\right)}{\rho_{0}^{2}}$.

Proof. This is obvious by the previous conclusions in this section.

Remark 3.1. If $g\left(\rho_{0}\right)=0$, then although we may use conclusion (i) in Theorem 3.4, it follows directly that $\lambda_{C}=-\frac{B\left(\rho_{0}\right)}{\rho_{0}^{2}}$. In other words, as far as the value of $\lambda_{C}$ is concerned, the conclusion agrees with situation (ii) when $g\left(\rho_{0}\right)=0$.

In applying Theorem 3.4, we need to calculate $g\left(\rho_{0}\right)$ which may not be an easy job for all cases. The reason is that $\rho_{0}$ may equal $q_{L}$ which is the largest zero of $B(s)$, and in many cases, it may be a hard job to calculate the exact value of $q_{L}$. Fortunately, this difficulty can be avoided. Indeed, note that in applying Theorem 3.4, the only thing we need to check is the sign of $g\left(\rho_{0}\right)$ rather than the exact value of $g\left(\rho_{0}\right)$ itself. However, as revealed by Lemma 3.3 , to check the sign of $g\left(\rho_{0}\right)$ is really a very easy job since all the conditions involved only concern with the quantity $\rho$ rather than $\rho_{0}$, as the following theorem shows. This tells us that in practical cases, we first use Lemma 3.3 to check the test function $g(s)$ and then apply Theorem 3.4 to get the decay parameter $\lambda_{C}$ together with $s_{*}$. Considering that in nearly all the practical situations, we are interested in tackling the conservative case, we present the following conclusion to summarize our basic results regarding determining 
the decay parameter of the stopped BABS queue, particularly for the conservative case. Recall $m_{d}$ and $m_{b}$ are defined in (1.6) and $\rho \geq 1$.

Theorem 3.5. If $\rho=+\infty$, then $g\left(\rho_{0}\right) \geq 0$, while if $\rho<+\infty$, then $g\left(\rho_{0}\right) \geq 0$ if and only if $g(\rho) \geq 0$. In particular, suppose that the stopped BABS generator $Q$ is conservative, then

(i) If $m_{b}>m_{d}$ (including $m_{b}=+\infty$ ), then $\lambda_{C}$ and $s_{*}$ can be obtained by using (3.31) or (3.32) and for this case, we have $0<s_{*}<1$ and $\lambda_{C}>0$.

(ii) If $m_{b}=m_{d}$, then $s_{*}=1$ and $\lambda_{C}=0$.

(iii) Assume that $m_{b}<m_{d}$.

(a) If $\rho=+\infty$, or if $1<\rho<+\infty$ and $0 \leq B(\rho) \leq+\infty$, then $\lambda_{C}$ and $s_{*}$ can be obtained by using (3.31) or (3.32) and that for this case, we have $1<s_{*}<+\infty$ and $\lambda_{C}>0$.

(b) If $\rho=1$, then $s_{*}=1$ and $\lambda_{C}=0$.

(c) Suppose that $1<\rho<+\infty$ and $B(\rho)<0$. Then if $B^{\prime}(\rho) \geq 0$ or if $B^{\prime}(\rho)<0$ with $\rho B^{\prime}(\rho) \geq 2 B(\rho)$, then $\lambda_{C}$ and $s_{*}$ can be determined by using (3.31) or (3.32) and that $1<s_{*} \leq \rho$ (with $s_{*}=\rho$ if and only if $\rho B^{\prime}(\rho)=2 B(\rho)$ ) and $\lambda_{C}>0$ while if $B^{\prime}(\rho)<0$ and $\rho B^{\prime}(\rho)<2 B(\rho)$, then $s_{*}=\rho$ and $\lambda_{C}=-\frac{B^{\prime}(\rho)}{2 \rho}$.

Proof. If $\rho=+\infty$, then by Lemma 3.3 we immediately get that $g\left(\rho_{0}\right) \geq 0$. Now we assume $\rho<+\infty$. If $B(\rho)>0$, then again by Lemma 3.3 we still automatically have $g\left(\rho_{0}\right) \geq 0$. However, by the proof of Lemma 2.3, we know that $g\left(\rho_{0}\right) \geq 0 \operatorname{implies} g(\rho) \geq 0$ and thus for this case the conclusion still holds. If $B(\rho) \leq 0$, then $\rho_{0}=\rho$, the conclusion holds trivially. Hence the first part of Theorem 3.5 is proven.

To prove the latter part, again, recall that $B(s)=0$ has either one positive root $q_{S}$ or has exactly two positive roots $q_{S} \leq q_{L}$. Now note that when $Q$ is conservative then $B^{\prime}(1)=m_{b}-m_{d}$ and thus, if $B^{\prime}(1)>0$ then $q_{S}<1$ and $q_{L}=1$ and if $B^{\prime}(1)=0$ then $q_{S}=q_{L}=1$. Moreover, if $B^{\prime}(1)<0$, then $q_{S}=1$ and there exists $q_{L}>1$ if and only if either $\rho=+\infty$ or $1<\rho<+\infty$ together with $0 \leq B(\rho) \leq+\infty$. Therefore, the conclusion follows from Lemma 3.3 and Theorem 3.4.

We shall see that it is extremely easy to determine the decay parameter by applying this theorem. In Section 6, we present examples to show that how easily these conclusions could be applied.

\section{Transience Properties}

Having obtained the decay parameter of the stopped BABS queueing process, we now further consider the $\lambda_{C}$-transience properties of the process. From now on, we shall assume that the stopped BABS generator $Q$ is conservative.

Lemma 4.1. Suppose that for some $\lambda>0$, the equation $B(s)+\lambda s^{2}=0$ has a positive root, $u(\lambda)>0$, say. Then for this fixed $\lambda>0, B(s)+\lambda s^{2}=0$ also has a negative root, denoted by $v(\lambda)$. Moreover, we have that $|v(\lambda)|<u(\lambda)$.

Proof. Since $B(u(\lambda))+\lambda u(\lambda)^{2}=0$, then by letting $s_{1}=-u(\lambda)$, we obtain

$$
B\left(s_{1}\right)+\lambda s_{1}^{2}=B(u(\lambda))+\lambda u(\lambda)^{2}-2 \sum_{j=0}^{\infty} b_{2 j+1} u(\lambda)^{2 j+1}=-2 \sum_{j=0}^{\infty} b_{2 j+1} u(\lambda)^{2 j+1}<0
$$

where the last inequality follows from (1.5). But $B(0)+\lambda 0^{2}=b_{0}>0$ and thus $B(s)+\lambda s^{2}=0$ possesses a negative root $v(\lambda) \in(-u(\lambda), 0)$. The fact $|v(\lambda)|<u(\lambda)$ also easily follows. 
In the following we shall use $u(\lambda)$ and $v(\lambda)$ to denote the smallest positive root and the largest negative root of $B(s)+\lambda s^{2}=0$, respectively. Note that for $\lambda<0$, we have used $u(\lambda)$ and $v(\lambda)$ to denote these two roots of $B(s)+\lambda s^{2}=0$, see Chen et al [8].

Lemma 4.2. Let $u(\lambda)$ and $v(\lambda)$ be the smallest positive and the largest negative root, respectively, of the equation $B(s)+\lambda s^{2}=0$ where $\lambda \in\left(-\infty, \lambda_{C}\right]$.

(i) $u(\lambda), v(\lambda) \in C^{\infty}\left(-\infty, \lambda_{C}\right)$.

(ii) $u(\lambda)$ is a decreasing function while $v(\lambda)$ is an increasing function of $\lambda \in\left(-\infty, \lambda_{C}\right)$.

(iii) $\lim _{\lambda \rightarrow 0} u(\lambda)=q_{S}$ and $\lim _{\lambda \rightarrow 0} v(\lambda)=\zeta$ where $q_{S}$ and $\zeta$ are the smallest positive root and the largest negative root, respectively, of the equation $B(s)=0$.

(iv) $\lim _{\lambda \rightarrow \lambda_{C}} u(\lambda)=s_{*}$ and $\lim _{\lambda \rightarrow \lambda_{C}} v(\lambda)=\zeta_{*}$ where $s_{*}$ and $\zeta_{*}$ are the smallest positive root and the largest negative root, respectively, of $B(s)+\lambda_{C} s^{2}=0$.

Proof. All conclusions follow from elementary considerations.

Remark 4.1. If one compares Lemma 4.2 with Lemma 3 in Chen et al [8], one would find, interestingly, that the two functions $u(\lambda)$ and $v(\lambda)$ defined here can be viewed as extensions of the two corresponding functions, bearing the same title in [8], with the domain extended from $(-\infty, 0)$ to $\left(-\infty, \lambda_{C}\right)$. Hence they inherit the relevant properties stated in Lemma 3 in Chen et al [8].

Lemma 4.3. Let $P(t)=\left(p_{i j}(t) ; i, j \in \mathbf{Z}_{+}\right)$be the stopped BABS-process (the $Q$-function) with generator matrix $Q$ as defined in (1.2)-(1.3). Then for any $i \geq 2$,

$$
\sum_{j=0}^{\infty} p_{i j}^{\prime}(t) s^{j}=B(s) \sum_{k=2}^{\infty} p_{i k}(t) s^{k-2}, \quad|s|<\rho_{0} .
$$

Proof. It is well-known (see Anderson [1]) that the corresponding $Q$-resolvent $\left(\phi_{i j}(\lambda) ; i, j \in\right.$ $\mathbf{Z}_{+}$) can be obtained by the forward recursion scheme

$$
\left\{\begin{array}{l}
\phi_{i j}^{(0)}(\lambda)=\frac{\delta_{i j}}{\lambda+q_{j}} \\
\phi_{i j}^{(n+1)}(\lambda)=\frac{\delta_{i j}}{\lambda+q_{j}}+\sum_{k \neq j} \phi_{i k}^{(n)}(\lambda) \frac{q_{k j}}{\lambda+q_{j}}, \quad n \geq 0
\end{array}\right.
$$

and $\phi_{i j}^{(n)}(\lambda) \uparrow \phi_{i j}(\lambda)$ as $n \uparrow \infty$ for all $i, j \in \mathbf{Z}_{+}$.

This recursion scheme together with mathematical induction immediately yields that for any $n \geq 0, i \geq 0$ and $0 \leq s \leq \rho_{0}$,

$$
\sum_{k=1}^{\infty} \phi_{i k}^{(n)}(\lambda) s^{k-1}<+\infty
$$

Also, by the above recursion scheme and (1.2), we know that for $|s| \leq \rho_{0}$,

$$
\sum_{j=0}^{\infty}\left(\lambda+q_{j}\right) \phi_{i j}^{(n+1)}(\lambda) s^{j}=s^{i}+\sum_{k=2}^{\infty} \phi_{i k}^{(n)}(\lambda) s^{k-2} \cdot\left(b_{0}+b_{1} s+\sum_{m=3}^{\infty} b_{m} s^{m}\right) .
$$

Noting that $\lambda+q_{j}=\lambda-b_{2}>-b_{2}(j \geq 2)$ and

$$
b_{0}+b_{1} s+\sum_{m=3}^{\infty} b_{m} s^{m} \leq b_{0}+b_{1} \rho_{0}+\sum_{m=3}^{\infty} b_{m} \rho_{0}^{m}
$$


for $s \in\left[0, \rho_{0}\right]$, we know that for $s \in\left[0, \rho_{0}\right]$,

$$
-b_{2} \sum_{j=2}^{\infty} \phi_{i j}^{(n+1)}(\lambda) s^{j} \leq s^{i}+\left(b_{0}+b_{1} \rho_{0}+\sum_{m=3}^{\infty} b_{m} \rho_{0}{ }^{m}\right) \sum_{k=2}^{\infty} \phi_{i k}^{(n)}(\lambda) s^{k-2}<+\infty .
$$

Now if we define $A_{i j}^{(n+1)}(\lambda)=\phi_{i j}^{(n+1)}(\lambda)-\phi_{i j}^{(n)}(\lambda) \quad(n \geq 0)$. Then $A_{i j}^{(n)}(\lambda) \geq 0$ and

$$
\lim _{n \rightarrow \infty} A_{i j}^{(n)}(\lambda)=0 \quad \text { for all } i, j \in \mathbf{Z}_{+} .
$$

Using this notation, (4.3) can be rewritten as

$$
\lambda \sum_{j=0}^{\infty} \phi_{i j}^{(n+1)}(\lambda) s^{j}=s^{i}+B(s) \sum_{k=2}^{\infty} \phi_{i k}^{(n)}(\lambda) s^{k-2}+b_{2} s^{2} \sum_{j=2}^{\infty} A_{i j}^{(n+1)}(\lambda) s^{j-2} .
$$

Letting $s=\rho_{0}$ in (4.4) we get that

$$
\sum_{k=2}^{\infty} A_{i k}^{(n)}(\lambda) \rho_{0}{ }^{k} \leq\left(-b_{2}\right)^{-1} \rho_{0}{ }^{i}, \quad n \geq 1
$$

Hence applying the Dominated Convergence Theorem and using (4.5) we obtain that for $s \in\left(0, \rho_{0}\right)$

$$
\lim _{n \rightarrow \infty} \sum_{j=2}^{\infty} A_{i j}^{(n+1)}(\lambda) s^{j-2}=0 .
$$

Letting $n \uparrow \infty$ in (4.6) and using the above limit yields that

$$
\lambda \sum_{j=0}^{\infty} \phi_{i j}(\lambda) s^{j}=s^{i}+B(s) \sum_{k=2}^{\infty} \phi_{i k}(\lambda) s^{k-2}, \quad s \in\left(0, \rho_{0}\right) .
$$

We now prove that both sides of (4.7) are finite for all $s \in\left[0, \rho_{0}\right)$. Indeed, this is trivial if $\rho_{0}=1$. If $\rho_{0}>1$ then we may find an $\varepsilon>0$ such that $B(s)<0$ for all $s \in\left(\rho_{0}-\varepsilon, \rho_{0}\right)$. Thus both sides of (4.7) must be finite for $s \in\left(\rho_{0}-\varepsilon, \rho_{0}\right)$ and hence must be also finite for all $s \in\left[0, \rho_{0}\right)$. It follows from (4.3) that (4.7) actually holds for all $|s|<\rho_{0}$.

By noting that the finite expression $\sum_{k=2}^{\infty} \phi_{i k}(\lambda) s^{k-2}$ is the Laplace transform of $\sum_{k=2}^{\infty} p_{i k}(t) s^{k-2}$, we know that $\sum_{k=2}^{\infty} p_{i k}(t) s^{k-2}<\infty$ for all $|s|<\rho_{0}$ and for almost all $t \geq 0$. Now, since for any $\lambda>0$ and $|s|<\rho_{0}$ we have $\lim _{t \rightarrow \infty} e^{-\lambda t} \sum_{k=2}^{\infty} p_{i k}(t)|s|^{k-2}=0$, it is easy to see that

$$
\lim _{t \rightarrow \infty} e^{-\lambda t} \int_{0}^{t}\left(\sum_{k=2}^{\infty} p_{i k}(u) s^{k-2}\right) d u=0
$$

Therefore, (4.7) can be rewritten as

$$
\int_{0}^{\infty} e^{-\lambda t}\left(\sum_{j=0}^{\infty} p_{i j}(t) s^{j}\right) d t=\frac{s^{i}}{\lambda}+B(s) \int_{0}^{\infty} e^{-\lambda t}\left\{\int_{0}^{t}\left(\sum_{k=2}^{\infty} p_{i k}(u) s^{k-2}\right) d u\right\} d t, \quad|s|<\rho_{0} .
$$


Hence,

$$
\sum_{j=0}^{\infty} p_{i j}(t) s^{j}=s^{i}+B(s) \int_{0}^{t}\left(\sum_{k=2}^{\infty} p_{i k}(u) s^{k-2}\right) d u, \quad|s|<\rho_{0}
$$

for almost all $t \geq 0$. However, the right hand side of the above equality is a differentiable function with respect to $t$, and thus we obtain

$$
\frac{\partial}{\partial t}\left(\sum_{j=0}^{\infty} p_{i j}(t) s^{j}\right)=B(s) \sum_{k=2}^{\infty} p_{i k}(t) s^{k-2}, \quad|s|<\rho_{0} .
$$

We now claim that

$$
\frac{\partial}{\partial t}\left(\sum_{j=0}^{\infty} p_{i j}(t) s^{j}\right)=\sum_{j=0}^{\infty} p_{i j}^{\prime}(t) s^{j}, \quad|s|<\rho_{0} .
$$

Indeed, by the Kolmogorov forward equations, we have

$$
p_{i j}^{\prime}(t)=\sum_{k=2}^{j+2} p_{i k}(t) b_{j-k+2}
$$

Now since $\sum_{k=2}^{\infty} p_{i k}(t) s^{k-2}<\infty$ and $B(s)<\infty$ for all $|s|<\rho_{0}$, we may get from the above equality that

$$
\sum_{j=0}^{\infty} p_{i j}^{\prime}(t) s^{j}=B(s) \sum_{k=2}^{\infty} p_{i k}(t) s^{k-2}, \quad|s|<\rho_{0} .
$$

The proof is complete.

We are now ready to consider whether the stopped BABS- process is $\lambda_{C}$-transient or not.

Theorem 4.1. Let $P(t)=\left(p_{i j}(t) ; i, j \in \mathbf{Z}_{+}\right)$be the stopped BABS-process with generator matrix $Q$ as defined in (1.2)-(1.3). Then for any $\lambda \in\left(-\infty, \lambda_{C}\right]$ and $i \geq 2$

$$
\int_{0}^{\infty} e^{\lambda t} p_{i 0}^{\prime}(t) d t=\frac{u(\lambda) v(\lambda)^{i}-v(\lambda) u(\lambda)^{i}}{u(\lambda)-v(\lambda)}
$$

and

$$
\int_{0}^{\infty} e^{\lambda t} p_{i 1}^{\prime}(t) d t=\frac{u(\lambda)^{i}-v(\lambda)^{i}}{u(\lambda)-v(\lambda)}
$$

Proof. By (4.1), for any $\lambda \in\left(-\infty, \lambda_{C}\right), i \geq 2$ and $s \in\left[-\rho_{0}, \rho_{0}\right]$,

$$
e^{\lambda t} p_{i 0}^{\prime}(t)+e^{\lambda t} p_{i 1}^{\prime}(t) s+\sum_{k=2}^{\infty} e^{\lambda t} p_{i k}^{\prime}(t) s^{k}=B(s) \sum_{k=2}^{\infty} e^{\lambda t} p_{i k}(t) s^{k-2} .
$$

Then for any fixed $T>0$, we may obtain

$$
\int_{0}^{T} e^{\lambda t} p_{i 0}^{\prime}(t) d t+s \int_{0}^{T} e^{\lambda t} p_{i 1}^{\prime}(t) d t+\int_{0}^{T}\left(e^{\lambda t} \frac{\partial F_{i}(t, s)}{\partial t} d t\right) s^{2}=B(s) \int_{0}^{T} e^{\lambda t} F_{i}(t, s) d t
$$


where $F_{i}(t, s)=\sum_{k=2}^{\infty} p_{i k}(t) s^{k-2}$. Using integration by parts, we get

$$
\left(B(s)+\lambda s^{2}\right) \int_{0}^{T} e^{\lambda t} F_{i}(t, s) d t=\int_{0}^{T} e^{\lambda t} p_{i 0}^{\prime}(t) d t+s \int_{0}^{T} e^{\lambda t} p_{i 1}^{\prime}(t) d t+s^{2} e^{\lambda T} F_{i}(T, s)-s^{i}(4.10)
$$

Now, recall that for $\lambda \in\left(-\infty, \lambda_{C}\right]$, the equation $B(s)+\lambda s^{2}=0$ has two roots $u(\lambda)$ and $v(\lambda)$ and thus by letting $s=u(\lambda)$ and $v(\lambda)$, respectively, in (4.10), we see that for any $T>0$,

$$
\int_{0}^{T} e^{\lambda t} p_{i 0}^{\prime}(t) d t+u(\lambda) \int_{0}^{T} e^{\lambda t} p_{i 1}^{\prime}(t) d t+u(\lambda)^{2} e^{\lambda T} F_{i}(T, u(\lambda))=u(\lambda)^{i}
$$

and

$$
\int_{0}^{T} e^{\lambda t} p_{i 0}^{\prime}(t) d t+v(\lambda) \int_{0}^{T} e^{\lambda t} p_{i 1}^{\prime}(t) d t+v(\lambda)^{2} e^{\lambda T} F_{i}(T, v(\lambda))=v(\lambda)^{i} .
$$

We now claim that

$$
\lim _{T \rightarrow \infty} e^{\lambda T} F_{i}(T, u(\lambda))=\lim _{T \rightarrow \infty} e^{\lambda T} F_{i}(T, v(\lambda))=0 .
$$

To prove (4.13), it is sufficient to prove that the first limit in (4.13) is zero since the second one is controlled by the first one, see Lemma 4.1. Note first that for all $t \geq 0$, we have $p_{i 0}^{\prime}(t) \geq 0$ and $p_{i 1}^{\prime}(t) \geq 0$ and thus all the three terms in the left hand side of (4.11) are nonnegative and also the first two terms are increasing with $T>0$. Observing that the right hand side of (4.11) is independent of $T>0$, we know that the third term in the left hand side of (4.11) is decreasing with $T>0$. Therefore, all three terms have finite nonnegative limit when $T \rightarrow \infty$. Hence, letting $T \rightarrow \infty$ in (4.11) yields

$$
\int_{0}^{\infty} e^{\lambda t} p_{i 0}^{\prime}(t) d t+u(\lambda) \int_{0}^{\infty} e^{\lambda t} p_{i 1}^{\prime}(t) d t+u(\lambda)^{2} \lim _{T \rightarrow \infty} e^{\lambda T} F_{i}(T, u(\lambda))=u(\lambda)^{i} .
$$

We now further prove that the third limit on the left hand side of (4.14) is zero for all $\lambda<\lambda_{C}$. To this end, assume that $\lim _{T \rightarrow \infty} e^{\lambda T} F_{i}(T, u(\lambda))>0$ for some $\lambda<\lambda_{C}$. Since $u(\lambda)$ is increasing with $\lambda$ and thus for any $\tilde{\lambda} \in\left(\lambda, \lambda_{C}\right)$, we have $F_{i}(T, u(\tilde{\lambda})) \geq F_{i}(T, u(\lambda))$. It then follows that $\liminf _{T \rightarrow \infty} e^{\lambda T} F_{i}(T, u(\tilde{\lambda}))>0$. Thus since $\tilde{\lambda}>\lambda$, we must have $\lim _{T \rightarrow \infty} e^{\tilde{\lambda} T} F_{i}(T, u(\tilde{\lambda}))=+\infty$. However, (4.11) also holds for $\lambda=\tilde{\lambda}$ and thus

$$
\int_{0}^{\infty} e^{\lambda t} p_{i 0}^{\prime}(t) d t+u(\tilde{\lambda}) \int_{0}^{\infty} e^{\tilde{\lambda} t} p_{i 1}^{\prime}(t) d t+u(\tilde{\lambda})^{2} \lim _{T \rightarrow \infty} e^{\tilde{\lambda} T} F_{i}(T, u(\tilde{\lambda}))=u(\tilde{\lambda})^{i}
$$

which contradicts with $\lim _{T \rightarrow \infty} e^{\tilde{\lambda} T} F_{i}(T, u(\tilde{\lambda}))=+\infty$. Therefore, $\lim _{T \rightarrow \infty} e^{\lambda T} F_{i}(t, u(\lambda))=0$ and hence (4.13) is proven. It then follows from (4.11) that

$$
\int_{0}^{\infty} e^{\lambda t} p_{i 0}^{\prime}(t) d t+u(\lambda) \int_{0}^{\infty} e^{\lambda t} p_{i 1}^{\prime}(t) d t=u(\lambda)^{i}
$$

which implies that $\int_{0}^{\infty} e^{\lambda t} p_{i 0}^{\prime}(t) d t<+\infty$ and $\int_{0}^{\infty} e^{\lambda t} p_{i 1}^{\prime}(t) d<+\infty$. Hence, by (4.12) we know that

$$
\int_{0}^{\infty} e^{\lambda t} p_{i 0}^{\prime}(t) d t+v(\lambda) \int_{0}^{\infty} e^{\lambda t} p_{i 1}^{\prime}(t) d t=v(\lambda)^{i}
$$

Now, (4.3) and (4.4) follow from (4.15) and (4.16) for the case of $\lambda<\lambda_{C}$.

Finally, by taking the appropriate limit, it can be easily proved that (4.8) and (4.9) also hold for $\lambda=\lambda_{C}$. 
Remark 4.2. Again, if one compares expressions (4.8) and (4.9) with expressions (26) and (27) presented in Theorem 1 in Chen et al [8], one would interestingly find, once again, that the former two expressions are just the extensions of the latter two to the domain $\left(-\infty, \lambda_{C}\right)$.

Theorem 4.2. Let $P(t)=\left(p_{i j}(t) ; i, j \in \mathbf{Z}_{+}\right)$be the stopped BABS-process with generator matrix $Q$ as defined in (1.2)-(1.3). Then for any $\lambda \in\left(-\infty, \lambda_{C}\right]$ and $i \geq 2$,

$$
\begin{aligned}
& \sum_{k=2}^{\infty}\left(\int_{0}^{\infty} e^{\lambda t} p_{i k}(t) d t\right) s^{k-2} \\
= & \frac{1}{B(s)+\lambda s^{2}}\left(\frac{u(\lambda) v(\lambda)^{i}-v(\lambda) u(\lambda)^{i}}{u(\lambda)-v(\lambda)}+\frac{u(\lambda)^{i}-v(\lambda)^{i}}{u(\lambda)-v(\lambda)} s-s^{i}\right), \quad|s|<u(\lambda)
\end{aligned}
$$

and, in particular,

$$
\int_{0}^{\infty} e^{\lambda t} p_{2 k}(t) d t=u(\lambda)^{4-k} \frac{G_{\lambda}^{(k-2)}(0)}{(k-2) !}, \quad(k \geq 2)
$$

where $G_{\lambda}^{(k)}(0)$ denotes the $k$ 'th derivative of

$$
G_{\lambda}(s)=\frac{1}{B(u(\lambda) s)+\lambda u(\lambda)^{2} s^{2}}\left[-\frac{v(\lambda)}{u(\lambda)}+\left(1+\frac{v(\lambda)}{u(\lambda)}\right) s-s^{2}\right]
$$

evaluated at 0. Hence the stopped BABS-process is always $\lambda_{C}$-transient.

Proof. Letting $T \rightarrow \infty$ in (4.10) and using (4.8)-(4.9) immediately yields (4.17). Then (4.18) follows directly from (4.17) by first letting $i=2$ and then letting $s=u(\lambda) s$.

\section{Invariant measures and Quasi-stationary Distributions}

We now turn our attention to the $\lambda_{C}$-invariant measures and quasi-stationary distributions for $P(t)$ on $C$. We shall assume in this section that $Q$ is conservative.

Theorem 5.1. Suppose that the generator matrix $Q$ defined in (1.2)-(1.3) is conservative. Let $P(t)=\left(p_{i j}(t) ; i, j \in \mathbf{Z}_{+}\right)$be the $Q$-function of the stopped BABS-process and $\lambda_{C}$ be the decay parameter of $C$. Then for any $\lambda \in\left[0, \lambda_{C}\right]$,

(i) there exists a $\lambda$-invariant measure $\left(m_{i} ; i \in C\right)$ for $Q$ on $C$, which is unique up to constant multiples. Moreover, the generating function of this $\lambda$-invariant measure $M(s)=\sum_{i=2}^{\infty} m_{i} s^{i-2}$ takes the simple form as

$$
M(s)=\frac{m_{2} b_{0}}{B(s)+\lambda s^{2}}\left(1-\frac{s}{v(\lambda)}\right), \quad|s|<u(\lambda)
$$

where $v(\lambda)$ is the largest negative root of $B(s)+\lambda s^{2}=0$ and $m_{2}>0$ is a constant.

(ii) This measure $\left(m_{i} ; i \in C\right)$ is also a $\lambda$-invariant measure for $P(t)$ on $C$.

(iii) This $\lambda$-invariant measure is convergent (i.e., $\left.\sum_{i \in C} m_{i}<\infty\right)$ if and only if $B^{\prime}(1)<0$, $\rho>1$ (including $\rho=+\infty$ ) and $0<\lambda \leq \lambda_{C}$, where $\rho$ is the convergence radius of $B(s)$. 
Proof. For $\lambda \in\left[0, \lambda_{C}\right]$, let $u(\lambda)$ and $v(\lambda)$ denote the smallest positive and the largest negative root of $B(s)+\lambda s^{2}=0$, respectively. Consider

$$
\widetilde{B}(s)=B(u(\lambda) s)+\lambda u(\lambda)^{2} s^{2}, \quad|s| \leq 1 .
$$

It is easy to see that $\widetilde{B}(1)=0$ and $\widetilde{B}^{\prime}(1) \leq 0$. By Lemma 2 of Chen et al [9], the function $G(s)=\frac{1-s}{\widetilde{B}(s)}\left(s-\frac{v(\lambda)}{u(\lambda)}\right)$ is well defined at least in $(-1,1)$ and can be expanded as a Taylor series

$$
G(s)=\sum_{k=0}^{\infty} g_{k} s^{k}, \quad|s|<1
$$

where the coefficients $g_{k}=G^{(k)}(0) / k !(k \geq 0)$ satisfy $0<g_{k} \leq g_{0}=-\frac{v(\lambda)}{b_{0} u(\lambda)}(k \geq 1)$. Therefore

$$
\frac{1}{\widetilde{B}(s)}\left(s-\frac{v(\lambda)}{u(\lambda)}\right)=\sum_{n=0}^{\infty}\left(\sum_{k=0}^{n} g_{k}\right) s^{n}, \quad|s|<1 .
$$

Choose $m_{2}>0$ and define

$$
m_{i+2}=\frac{m_{2}}{g_{0}(u(\lambda))^{i}} \sum_{k=0}^{i} g_{k}, \quad i \geq 1 .
$$

Then the sequence $\left\{m_{j} ; j \geq 2\right\}$ so defined is clearly strictly positive. Now for all $|s|<u(\lambda)$,

$$
\begin{aligned}
M(s) & =\sum_{i=0}^{\infty} m_{i+2} s^{i}=\frac{m_{2}}{g_{0}} \sum_{i=0}^{\infty}\left(\sum_{k=0}^{i} g_{k}\right)\left(\frac{s}{u(\lambda)}\right)^{i} \\
& =\frac{m_{2}}{g_{0} \widetilde{B}\left(\frac{s}{u(\lambda)}\right)}\left(\frac{s}{u(\lambda)}-\frac{v(\lambda)}{u(\lambda)}\right) \\
& =\frac{m_{2}}{g_{0}\left(B(s)+\lambda s^{2}\right)}\left(\frac{s}{u(\lambda)}-\frac{v(\lambda)}{u(\lambda)}\right) \\
& =\frac{m_{2} b_{0}}{B(s)+\lambda s^{2}}\left(1-\frac{s}{v(\lambda)}\right) .
\end{aligned}
$$

Here the last equality follows from the fact that $g_{0}=-\frac{v(\lambda)}{b_{0} u(\lambda)}$. Hence

$$
B(s) M(s)-m_{2} b_{0}\left(1-\frac{s}{v(\lambda)}\right)=-\lambda M(s) s^{2}, \quad|s|<u(\lambda) .
$$

Comparing the coefficient of $s$ in the above equality, we know that the coefficient of $s$ in the left-hand side must be 0 , i.e,

$$
m_{3} b_{0}+m_{2} b_{1}+\frac{m_{2} b_{0}}{v(\lambda)}=0
$$

Therefore

$$
B(s) M(s)-m_{2} b_{0}-\left(m_{3} b_{0}+m_{2} b_{1}\right) s=-\lambda M(s) s^{2}, \quad|s|<u(\lambda)
$$


and hence

$$
\sum_{j=2}^{\infty}\left(\sum_{i=2}^{j+2} m_{i} b_{j-i+2}\right) s^{j}=-\lambda M(s) s^{2}, \quad|s|<u(\lambda) .
$$

Noting that $M(s)=\sum_{j=2}^{\infty} m_{j} s^{j-2}$, we get

$$
\sum_{i=2}^{j+2} m_{i} b_{j-i+2}=-\lambda m_{j}, \quad j \geq 2
$$

which shows that $\left(m_{i} ; i \in C\right)$ is a $\lambda$-invariant measure for $Q$ on $C$.

We now prove uniqueness. Suppose that $\mu \in\left[0, \lambda_{C}\right]$ and $\left(m_{i} ; i \geq 2\right)$ is a $\mu$-invariant measure for $Q$ on $C$, that is, $m_{i}>0(i \geq 2)$ and

$$
\sum_{i=2}^{j+2} m_{i} b_{j-i+2}=-\mu m_{j}, \quad j \geq 2 .
$$

Let $b=-b_{2}$, then $b>0$ and $b>\lambda_{C}$. We shall prove the uniqueness in three steps. First show that the generating function $M(s)=\sum_{j=2}^{\infty} m_{j} s^{j-2}$ of $\left\{m_{j} ; j \geq 2\right\}$ is well defined at least on $\left(-\sqrt{\frac{b_{0}}{b-\mu}}, \sqrt{\frac{b_{0}}{b-\mu}}\right)$. Indeed, note that (5.4) can be rewritten, after replacing $b_{2}=-b$, as

$$
m_{j+2} b_{0}=(b-\mu) m_{j}-\sum_{i \in\{2, \cdots, j+1\} \backslash\{j\}} m_{i} b_{j-i+2}, \quad j \geq 2
$$

and hence $m_{j+2} \leq \frac{b-\mu}{b_{0}} m_{j}$. Therefore,

$$
\begin{gathered}
m_{2 j} \leq\left(\frac{b-\mu}{b_{0}}\right)^{j-1} m_{2}, \quad j \geq 2 \\
m_{2 j+1} \leq\left(\frac{b-\mu}{b_{0}}\right)^{j-1} m_{3}, \quad j \geq 2 .
\end{gathered}
$$

It follows from (5.6) and (5.7) that the convergence radius of $M(s)=\sum_{j=2}^{\infty} m_{j} s^{j-2}$ is at least $\sqrt{\frac{b_{0}}{b-\mu}}$.

Secondly, it follows from (5.4) that for all $s \in\left(-\sqrt{\frac{b_{0}}{b-\mu}}, \sqrt{\frac{b_{0}}{b-\mu}}\right)$,

$$
\sum_{j=2}^{\infty}\left(\sum_{i=2}^{j+2} m_{i} b_{j-i+2}\right) s^{j}=-\mu \sum_{j=2}^{\infty} m_{j} s^{j}
$$

Using some algebra yields that for all $s \in\left(-\sqrt{\frac{b_{0}}{b-\mu}}, \sqrt{\frac{b_{0}}{b-\mu}}\right)$,

$$
\left(B(s)+\mu s^{2}\right) M(s)=m_{2} b_{0}+\left(m_{3} b_{0}+m_{2} b_{1}\right) s .
$$


Note that although (5.9) is obtained under the condition that $|s|<\sqrt{\frac{b_{0}}{b-\mu}}$, the right hand side of (5.9) is positive for all $s>0$. However, $B(s)+\mu s^{2}$ is finite and in fact positive for all $s \in(0, u(\mu))$, where $u(\mu)$ is the smallest positive zero of $B(s)+\mu s^{2}$ as defined above, and thus $M(s)$ is also well-defined. That is that $M(s)$ is finite for all $s \in(0, u(\mu))$. Furthermore, we have

$$
M(s)=\frac{m_{2} b_{0}+\left(m_{3} b_{0}+m_{2} b_{1}\right) s}{B(s)+\mu s^{2}}, \quad s \in(0, u(\mu)) .
$$

Recall that $B(s)+\mu s^{2}=0$ has a largest negative root $v(\mu) \in(-u(\mu), 0)$ and thus $M(v(\mu))$ is finite. It then follows from (5.9) that

$$
m_{2} b_{0}+\left(m_{3} b_{0}+m_{2} b_{1}\right) v(\mu)=0 .
$$

Noting that $B(s)+\mu s^{2}=0$ has exactly two roots on $[-u(\mu), u(\mu)]$, we may further write (5.10) as

$$
M(s)=\frac{m_{2} b_{0}+\left(m_{3} b_{0}+m_{2} b_{1}\right) s}{B(s)+\mu s^{2}}, \quad s \in(-u(\mu), u(\mu)) .
$$

Because of (5.11), we know that the $\mu$-invariant measure is unique up to constant multiples.

We now prove (ii). By Theorem 5.4.3 of Anderson [1], we only need to show that the equations

$$
\sum_{i=2}^{j+2} y_{i} b_{j-i+2}=-\nu y_{j}, \quad 0 \leq y_{j} \leq m_{j}, \quad j \geq 2
$$

has no nontrivial solution $\left(y_{i} ; i \geq 2\right)$ for some $\nu<\lambda$. Indeed, suppose that $\left(y_{i} ; i \geq 2\right)$ is a solution of (5.13). Since $|M(s)|<\infty$ for all $|s|<u(\lambda)$ and $y_{j} \leq m_{j}(j \geq 2)$, we know that $Y(s)=\sum_{j=2}^{\infty} y_{j} s^{j-2}$ is convergent for all $|s|<u(\lambda)$. Hence, it is easily seen that for $|s|<u(\lambda)$,

$$
\left(B(s)+\nu s^{2}\right) Y(s)=y_{2} b_{0}+\left(y_{3} b_{0}+y_{2} b_{1}\right) s .
$$

Since $\nu<\lambda$, we know that $B(s)+\nu s^{2}=0$ has a positive root $\widetilde{s} \in(0, u(\lambda))$. Letting $s=\widetilde{s}$ in the above equality and noting $Y(s) \leq M(s)<\infty$ for all $s \in(0, u(\lambda))$ yields that $y_{2}=y_{3}=0$. Hence, $Y(s)=0$ for $s \in(0, u(\lambda))$ which implies that $y_{i}=0(i \geq 2)$. Conclusion(ii) is thus proven.

Finally, note that $B(s)+\lambda s^{2}=0$ has a smallest positive root $u(\lambda)>1$ if and only if $B^{\prime}(1)<0, \rho>1$ and $0<\lambda \leq \lambda_{C}$ and thus conclusion (iii) follows from (5.1). The proof is complete.

Remark 5.1. Since a $\lambda$-invariant measure for $P(t)$ on $C$ must be a $\lambda$-invariant measure for $Q$ on $C$. Theorem 5.1 implies that the $\lambda$-invariant measure for $P(t)$ on $C$ is unique up to constant multiples.

Having given the $\lambda_{C}$-invariant measure, we now further consider the quasi-stationary distributions for $P(t)=\left(p_{i j}(t) ; i, j \in \mathbf{Z}_{+}\right)$on $C$. 
Theorem 5.2. Suppose that the generator matrix $Q$ defined in (1.2)-(1.3) is conservative. Let $P(t)=\left(p_{i j}(t) ; i, j \in \mathbf{Z}_{+}\right)$be the corresponding stopped BABS queueing process and $\lambda_{C}$ be the decay parameter determined as above. Then there exists a quasi-stationary distribution for $P(t)=\left(p_{i j}(t) ; i, j \in \mathbf{Z}_{+}\right)$on $C$ if and only if $B^{\prime}(1)<0$ and $\rho>1$. Moreover, if these conditions hold, then there exist one-parameter family of quasi-stationary distributions $\left\{\left(m_{i}(\lambda) ; i \in C\right) ; \lambda \in\left(0, \lambda_{C}\right]\right\}$ which can be given by

$$
M_{\lambda}(s)=\frac{\lambda(s-v(\lambda))}{(1-v(\lambda))\left(B(s)+\lambda s^{2}\right)}, \quad|s|<u(\lambda)
$$

where $M_{\lambda}(s)=\sum_{i=2}^{\infty} m_{i}(\lambda) s^{i-2}$ and $v(\lambda)$ is the largest negative root of $B(s)+\lambda s^{2}=0$.

Proof. By Proposition 3.1 of Nair and Pollett [32], a probability distribution $\left(m_{i} ; i \in C\right)$ on $C$ is a quasi-stationary distribution for $P(t)=\left(p_{i j}(t) ; i, j \in \mathbf{Z}_{+}\right)$on $C$ if and only if, for some $\lambda>0,\left(m_{i} ; i \in C\right)$ is $\lambda$-invariant for $P(t)=\left(p_{i j}(t) ; i, j \in \mathbf{Z}_{+}\right)$on $C$. Thus the conclusions follow from Theorem 5.1.

\section{Examples}

In this section, we present some examples to illustrate the results obtained in the previous sections. Since a stopped BABS generator matrix only depends on the sequence $\left\{b_{k} ; k \geq 0\right\}$ and it is clear how to construct the generator matrix $Q$ from this sequence, we shall, in the following examples, only specify the sequence $\left\{b_{k} ; k \geq 0\right\}$ and then discuss the decay parameter $\lambda_{C}$ of the corresponding queueing process.

Example 6.1. Assume that $b_{0}=d_{2}>0, b_{1}=d_{1} \geq 0$ and there exists $k \geq 3$ such that $b_{k}=b>0$ and $b_{j}=0(j \geq k+1)$ and $b_{2}=-\left(d_{2}+d_{1}+\sum_{j=3}^{k} b_{j}\right)$. This is a queueing model that no more than $k-2$ customers (if $k=3$, then no more than 1 customer) will arrive at the queue and the serve rates for 1 and 2 customers are $d_{1}$ and $d_{2}$, respectively. For this example, we have $\rho=+\infty$ and

$$
B(s)=d_{2}+d_{1} s-\left(d_{2}+d_{1}+\sum_{j=3}^{k} b_{j}\right) s^{2}+\sum_{j=3}^{k} b_{j} s^{j}
$$

and

$$
B^{\prime}(s)=d_{1}-2\left(d_{2}+d_{1}+\sum_{j=3}^{k} b_{j}\right) s+\sum_{j=3}^{k} j b_{j} s^{j-1} .
$$

By Theorem 3.5, we know that $\lambda_{C}$ and $s_{*}$ can be determined by using (3.31) or (3.32). That is that $s_{*}$ is the positive zero of the polynomial

$$
\sum_{j=3}^{k}(j-2) b_{j} s^{j}-d_{1} s-2 d_{2}
$$

and then $\lambda_{C}=-\frac{B^{\prime}\left(s_{*}\right)}{2 s_{*}}$. In particular, if $k=3$ and $b_{3}=b>0$, then

$$
B(s)=d_{2}+d_{1} s-\left(d_{1}+d_{2}+b\right) s^{2}+b s^{3}
$$


and

$$
B^{\prime}(s)=d_{1}-2\left(d_{1}+d_{2}+b\right) s+3 b s^{2} .
$$

The equation (3.32) becomes

$$
\left\{\begin{array}{l}
b s^{3}-2 d_{2}-d_{1} s=0 \\
2 \lambda s+d_{1}-2\left(d_{1}+d_{2}+b\right) s+3 b s^{3}=0
\end{array}\right.
$$

If $d_{1}=0$, then

$$
\left\{\begin{array}{l}
b s^{3}-2 d_{2}=0 \\
2 \lambda s-2\left(d_{2}+b\right) s+3 b s^{3}=0
\end{array}\right.
$$

and hence the equation $b s^{3}-2 d_{2}=0$ has a unique positive root $s_{*}=\sqrt[3]{2 d_{2} / b}$ (the other two roots are complex). Therefore, we have

$$
\lambda_{C}=d_{2}+b-\frac{3}{2} \sqrt[3]{2 d_{2} b^{2}}
$$

If $d_{1}>0$, then the unique positive root of the equation $b s^{3}=2 d_{2}+d_{1} s$ is

$$
s_{*}=\sqrt[3]{\frac{d_{2}}{b}+\sqrt{\left(\frac{d_{2}}{b}\right)^{2}-\left(\frac{d_{1}}{3 b}\right)^{3}}}+\sqrt[3]{\frac{d_{2}}{b}-\sqrt{\left(\frac{d_{2}}{b}\right)^{2}-\left(\frac{d_{1}}{3 b}\right)^{3}}}
$$

and hence

$$
\lambda_{C}=\left(d_{1}+d_{2}+b\right)-\frac{3 b}{2} s_{*}^{2}-\frac{d_{1}}{2 s_{*}} .
$$

For any $\lambda \in\left[0, \lambda_{C}\right]$, the generating function of $\lambda$-invariant measure $M(s)=\sum_{i=2}^{\infty} m_{i} s^{i-2}$ is given by

$$
M(s)=\frac{m_{2} d_{2}}{B(s)+\lambda s^{2}}\left(1-\frac{s}{v(\lambda)}\right)
$$

where $v(\lambda)$ is the largest negative root of $B(s)+\lambda s^{2}=0$ and $m_{2}>0$ is a constant. This $M(s)$ gives a quasi-stationary distribution if and only if $\sum_{j=3}^{k}(j-2) b_{j}<2 d_{2}+d_{1}$. In particular, if $k=3$ and $b_{3}=b>0$, then this $M(s)$ gives a quasi-stationary distribution if and only if $b<2 d_{2}+d_{1}$.

Example 6.2. Let $b_{0}=(1-\alpha) a, b_{1}=(1-\alpha)(1-a), b_{j}=\alpha(1-b) b^{j-3}(j \geq 3)$ and $b_{2}=-1$ where $0<a \leq 1,0<\alpha<1,0 \leq b<1$. This is a queue that the arrival rates follow a geometric distribution coupled with the serve rates follow a Bernoulli distribution. For this example, we have

$$
B(s)=(1-\alpha)[a+(1-a) s]-s^{2}+\frac{\alpha(1-b) s^{3}}{1-b s} .
$$


Hence, $\rho=1 / b$ and $B(\rho)=+\infty$. Therefore by Theorem 3.5 we see that $\lambda_{C}$ and $s_{*}$ must satisfy Equation (3.32) in the unknowns $\lambda$ and $s$. That is

$$
\left\{\begin{array}{l}
s B^{\prime}(s)-2 B(s)=0 \\
\lambda=-\frac{B^{\prime}(s)}{2 s} .
\end{array}\right.
$$

Recall $g(s)=s B^{\prime}(s)-2 B(s)$, it is thus easy to see that

$$
g(s)=\frac{\alpha(1-b) s^{3}}{(1-b s)^{2}}-(1-\alpha)(2 a+(1-a) s) .
$$

Note that $g(0)=-2(1-\alpha) a<0$ and $g(1 / b)=+\infty$ and thus $g(s)=0$ has a root $s_{*}$ in $(0,1 / b)$. By Lemma 2.5 we also know that $s_{*}$ is the unique positive root of $g(s)=0$. Note also that $g(1)=\alpha(1-b)^{-1}-(1-\alpha)(1+a)$ and hence if $\alpha /(1-b)>(1-\alpha)(1+a)$ then $s_{*} \in(0,1)$ while if $\alpha /(1-b)<(1-\alpha)(1+a)$ then $s_{*} \in(1,1 / b)$. The above calculation coincides with our intuitive explanation. Indeed, for this example, $m_{b}=\alpha /(1-b)$ and $m_{d}=(1-\alpha)(1+a)$ and thus $m_{b}>m_{d}$ if and only if $\alpha /(1-b)>(1-\alpha)(1+a)$

To get the exact value of $s_{*}$, we need to find the positive root of the equation

$$
\alpha(1-\alpha)^{-1}(1-b) s^{3}=[2 a+(1-a) s](1-b s)^{2}
$$

or

$$
\left[\alpha(1-\alpha)^{-1}(1-b)-(1-a) b^{2}\right] s^{3}+2 b(1-a-a b) s^{2}+(4 a b+a-1) s-2 a=0
$$

After finding this positive root $s_{*}$, then

$$
\lambda_{C}=1-\frac{1}{2}\left[\frac{(1-\alpha)(1-a)}{s_{*}}+\alpha(1-b) s_{*} \frac{3-2 b s_{*}}{\left(1-b s_{*}\right)^{2}}\right] .
$$

In particular, if $a=1$ (that is $b_{0}=1-\alpha$ and $b_{1}=0$ ), then Equation (6.4) becomes

$$
\alpha(1-\alpha)^{-1}(1-b) s^{3}-2 b^{2} s^{2}+4 b s-2=0
$$

and then

$$
\lambda_{C}=1-\alpha(1-b) \frac{3 s_{*}-2 b s_{*}^{2}}{2\left(1-b s_{*}\right)^{2}}
$$

Note also that if $a=1$ then $g(1)=\frac{\alpha}{1-b}-2(1-\alpha)$ and hence if $\alpha(1-\alpha)^{-1}>2(1-b)$ then $0<s_{*}<1$ while if $\alpha(1-\alpha)^{-1}<2(1-b)$ then $1<s_{*}<1 / b$. In the special case of $\alpha(1-\alpha)^{-1}=2(1-b)$, we have $s_{*}=1$ and thus $\lambda_{C}=0$. For all other cases, $\lambda_{C}>0$.

For any $\lambda \in\left[0, \lambda_{C}\right]$, the generating function of $\lambda$-invariant measure $M(s)=\sum_{i=2}^{\infty} m_{i} s^{i-2}$ is given by

$$
M(s)=\frac{m_{2}(1-\alpha) a}{B(s)+\lambda s^{2}}\left(1-\frac{s}{v(\lambda)}\right)
$$

where $v(\lambda)$ is the largest negative root of $B(s)+\lambda s^{2}=0$ and $m_{2}>0$ is a constant. This $M(s)$ gives a quasi-stationary distribution if and only if $(1-\alpha)(1-a)+\frac{3 \alpha-2 b}{1-b}<2$. 
Finally, we consider a more subtle example. We shall see that even for this complicated example, Theorem 3.5 still works well.

Example 6.3. Suppose that $b_{0}=d_{2}>0, b_{1}=d_{1} \geq 0, b_{k}=\frac{b \theta^{k}}{k(k-1)(k-2)}(k \geq 3)$ and $b_{2}=-\left[d_{2}+d_{1}+b \sum_{k=3}^{\infty} \frac{\theta^{k}}{k(k-1)(k-2)}\right]$, where $b>0$ and $0<\theta \leq 1$.

For this example we have

$$
B(s)=d_{2}+d_{1} s+b_{2} s^{2}+b \sum_{k=3}^{\infty} \frac{(\theta s)^{k}}{k(k-1)(k-2)}
$$

and thus $\rho=1 / \theta<+\infty$. Easy algebra then yields

$$
B^{\prime}(s)=d_{1}+2 b_{2} s+b \sum_{k=3}^{\infty} \frac{\theta^{k} s^{k-1}}{(k-1)(k-2)}
$$

and

$$
g(s)=-2 d_{2}-d_{1} s+b \sum_{k=3}^{\infty} \frac{(\theta s)^{k}}{k(k-1)} .
$$

By (6.7), we immediately obtain

$$
g(\rho)=g\left(\frac{1}{\theta}\right)=\frac{b}{2}-\left(2 d_{2}+\frac{d_{1}}{\theta}\right)
$$

which is nonnegative if and only if $\frac{b}{2} \geq 2 d_{2}+\frac{d_{1}}{\theta}$.

Note also that closed forms for $B(s), B^{\prime}(s)$ and $g(s)$ in (6.5)-(6.7) are available. In particular, after easy algebra, $B^{\prime}(s)$ can be written as

$$
B^{\prime}(s)=d_{1}+\left[\frac{b \theta(2-\theta)}{2}+b(1-\theta)^{2} \ln (1-\theta)-2\left(d_{2}+d_{1}\right)\right] s+b \theta(1-\theta s) \ln (1-\theta s) .(6.8)
$$

The form of $B^{\prime}(s)$ in (6.8) also holds for $\theta=1$ under the convention that $\lim _{\theta \rightarrow 1}(1-\theta) \ln (1-$ $\theta)=0$.

Now, an application of Theorems 3.4 and 3.5 immediately yields the following conclusion.

Theorem 6.1. (a) Suppose $0<\theta<1$.

(i) If $\frac{b}{2} \geq 2 d_{2}+\frac{d_{1}}{\theta}$, then $\lambda_{C}=-\frac{B^{\prime}\left(s_{*}\right)}{2 s_{*}}$ where $B^{\prime}(s)$ is given in (6.8) and $s_{*}$ is the unique positive root of the equation

$$
(1-\theta s) \ln (1-\theta s)-\frac{\theta^{2} s^{2}}{2}+\left(\theta-\frac{d_{1}}{b}\right) s=\frac{2 d_{2}}{b} .
$$

(ii) If $\frac{b}{2}<2 d_{2}+\frac{d_{1}}{\theta}$, then

$$
\lambda_{C}=d_{2}+d_{1}\left(1-\frac{\theta}{2}\right)-\frac{b \theta(2-\theta)}{4}-\frac{b(1-\theta)^{2}}{2} \ln (1-\theta)
$$


(b) Suppose $\theta=1$. Then $\lambda_{C}=0$ if and only if $\frac{b}{2} \leq 2 d_{2}+d_{1}$ and for the case of $\frac{b}{2}>2 d_{2}+d_{1}$, this positive $\lambda_{C}$ is given by

$$
\lambda_{C}=d_{2}+d_{1}-\frac{b}{4}-\frac{d_{1}+b\left(1-s_{*}\right) \ln \left(1-s_{*}\right)}{2 s_{*}}
$$

where $0<s_{*}<1$ is the unique positive root of the equation

$$
(1-s) \ln (1-s)-\frac{s^{2}}{2}+\left(1-\frac{d_{1}}{b}\right) s=\frac{2 d_{2}}{b}
$$

Proof. Just note that $g(\rho) \geq 0$ if and only if $\frac{b}{2} \geq 2 d_{2}+\frac{d_{1}}{\theta}$ and that, by some easy algebra, $g\left(s_{*}\right)=0$ if and only if $s_{*}$ is the unique positive root of the Equation (6.9), then the conclusion in Part (a) immediately follows from Theorems 3.4 and 3.5. The conclusion in Part (b) is also easy. In particular, Equation (6.11) is just the version of Equation (6.9) when $\theta=1$. The proof is complete.

\section{Acknowledgements}

The authors would like to express their sincere thanks for the anonymous referees who have provided extremely helpful comments and suggestions, which led to a much improved presentation of this paper. The work described in this paper by Chen Anyue was substantially supported by a grant from the Research Grants Council of the Hong Kong Special Administrative Region, China, Project HKU 7010/06P and the work by Li Junping and Hou Zhenting was supported by the National Natural Science Foundation of China (grant No. 10771216) and Doctoral Fund of Ministry of Education of China (grant No. 20090162110058), respectively.

\section{References}

[1] Anderson,W. (1991) Continuous-Time Markov Chains: An Applications-Oriented Approach. Springer-Verlag, New York.

[2] Armero, C and Conesa, D. (2000) Prediction in Markovian bulk arrival queues. Queueing Systems Theory Appl. 34, 327-350.

[3] Arumuganathan, R. and Ramaswami, K.S. (2005) Analysis of a bulk queue with state dependent arrivals and multiple vacations. Indian J. Pure Appl. Math. 36, 301317.

[4] Asmussen,S. (2003) Applied Probability and Queues. 2nd ed. Springer-Verlag, New York.

[5] Bayer,N. And Boxma,O.J. (1996) Wiener-Hopf analysis of an M/G/1 queue with negative customers and of a related class of random walks. Queueing Systems 23, 301-316.

[6] Chang, S.H., Choi, D.W. And Kim, T.S. (2004) Performance analysis of a finitebuffer bulk-arrival and bulk-service queue with variable server capacity. Stochastic Anal. Appl. 22, 1151-1173. 
[7] Chaudhry,M.L. and Templeton,J.G.C. (1983) A First Course in Bulk Queues. Wiley: New York.

[8] Chen,A.Y., Pollett,P.K., Li,J.P And Zhang, H.J.(2010) Markovian bulk-arrival and bulk-service queues with state-dependent control. Queueing Systems, 64, 267-304.

[9] Chen,A.Y., Pollett,P, Li,J.P. And Zhang,H.J. (2010) Uniqueness, extinction and explosivity of generalised Markov branching processes with pairwise interaction. Methodol. Comput. Appl. Probab.(DOI:10.1007/s11009-009-9121-y).

[10] Chen, A.Y. And Renshaw,E. (1997) The M/M/1 queue with mass exodus and mass arrivals when empty. J. Appl. Probab., 34, 192-207.

[11] Chen,A.Y. and Renshaw,E. (2004) Markovian bulk-arriving queues with statedependent control at idle time. Adv. Appl. Probab. 36, 499-524.

[12] Chung,K.L. (1967) Markov Chains with Stationary Transition Probabilities. 2nd edn. Springer, New York.

[13] Darroch, J.N. AND Seneta, E. (1967) On quasi-stationary distributions in absorbing continuous-time finite Markov chains. J. Appl. Probab., 4, 192-196.

[14] Dudin,A. And Nishimura,S. (1999) A BMAP/SM/1 queueing system with Markovian arrival input of disasters. J. Appl. Probab., 36, 868-881.

[15] Fakinos, D. (1991) The relation between limiting queue size distributions at arrival and departure epochs in a bulk queue. Stochastic Process. Appl. 37, 327-329.

[16] Flaspohler, D.C.(1974) Quasi-stationary distributions for absorbing continuoustime denumerable Markov chains. Ann. Inst. Statist. Math. , 26, 351-356.

[17] Gelenbe,E. (1991) Product from networks with negative and positive customers. J. Appl. Probab., 28, 656-663.

[18] Gelenbe,E., Glynn, P. And Sigman (1991) Queues with negative arrivals. J. Appl. Probab., 28, 245-250.

[19] Gross, D. And Harris, C.M.(1985) Fundamentals of Queueing Theory. Wiley: New York

[20] Harrison,P.G. And Pitel,E. (1993) Sojourn times in single-server queues with negative customers. J. Appl. Probab., 30, 943-963.

[21] Henderson,W. (1993) Queueing networks with negative customers and negative queue lengths. J. Appl. Probab., 30, 931-942.

[22] Jain,G. And Sigman,K. (1996) A Pollaczek-Khintchine formula for M/G/1 queues with disasters. J. Appl. Probab., 33, 1191-1200.

[23] Kelly, F.P. (1983) Invariant measures and the generator. In Kingman, J.F.C. and Reuter, G.E., eds. Probability, Statistics, and Analysis. London Math. Soc. Lecture Notes Series 79. Cambridge University Press, pp. 143-160. 
[24] KiJima, M. (1963) Quasi-limiting distributions of Markov chains that are skip-free to the left in continuous-time. J. Appl. Probab., 30, 509-517.

[25] Kingman,J.F.C. (1963) The exponential decay of Markov transition probability. Proc. London Math. Soc., 13, 337-358.

[26] Kleinrock,I. (1975) Queueing Systems, Vol.1. Wiley: New York.

[27] Li, J.P. And Chen, A.Y. (2008) Decay property of stopped Markovian bulk-arriving queues. Adv. Appl. Probab., 40, 95-121.

[28] Li, J.P. And Chen, A.Y. (2010) Decay parameter and invariant measures for Markovian bulk-arrival queues with control at idle time. To appear in Methodology Comput. Appl. Prob.

[29] Lucantoni, D. M. (1991) New results on the single server queue with a batch Markovian arrival process. Stoch. Models, 7, 1-46.

[30] Lucantoni,D. M. And Neuts,M.F.(1994) Some steady-state distributions for the MAP/SM/1 queue. Stoch. Models , 10, 575-589.

[31] Medhi,J.(1991) Stochastic Models in Queuing Theory. Academic Press, San Diego, CA.

[32] Nair,M.G. AND Pollett,P.K.(1993) On the relationship between $\mu$-invariant measures and quasi-stationary distributions for continuous-time Markov chains. Adv. Appl. Probab. , 25, 82-102.

[33] Neuts,M.F.(1979) A versatile Markovian point process. J. Appl. Probab., 16, 764-779.

[34] Neuts, M. F.(1981) Matrix-Geometric Solution in Stochastic Models: An Algorithmic Approach. Johns Hopkins University Press, Baltimore, MD.

[35] Nishimura,S. And Sato,H.(1997) Eigenvalue expression for a batch Markovian arrival process. J. Operat. Res. Soc. Japan , 40, 122-132.

[36] Pollett,P.K.(1988) Reversibility, invariance and mu-invariance. Adv. Appl. Probab., 20, 600-621.

[37] Srinivasan, L., Renganathan, N. and Kalyanaraman, R. (2002) Single server, bulk arrival, Bernoulli feedback queue with vacations - some performance measures. Internat. J. Inform. Management Sci. 13, 45-54.

[38] Stadje,W.(1989) Some exact expressions for the bulk-arrival queue $M^{X} / M / 1$. Queuing Systems , 4, 85-92.

[39] Sumita, U. and Masuda, Y. (1997) Tandem queues with bulk arrivals, infinitely many servers and correlated service times. J. Appl. Probab. 34, 248-257.

[40] Ushakumari, P.V. and Krishnamoorthy, A. (1998) On a bulk arrival bulk service infinite server queue. Stochastic Anal. Appl. 16, 585-595. 
[41] Tweedie, R.L.(1974) Some ergodic properties of the Feller minimal process. Quart, J. Math. Oxford, (2)25, 485-493.

[42] VAn Doorn, E.A.(1985) Conditions for exponential ergodicity and bounds for the decay parameter of a birth-death process. Adv. Appl. Probab. , 17, 514-530.

[43] Van Doorn, E.A.(1991) Quasi-stationary distributions and convergence to quasistationarity of birth-death processes. Adv. Appl. Probab. , 23, 683-700.

[44] Vere-Jones, D.(1962) Geometric ergidicity in denumerable Markov chains. Quart, J. Math. Oxford, (2)13, 7-28.

[45] Yaglom, A.M.(1947) Certain limit theorems of the theory of branching processes. Dokl. Acad. Nauk SSSR, 56, 795-798. 